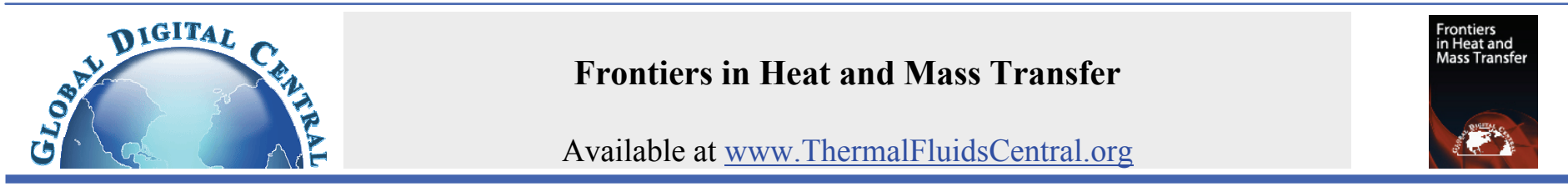

\title{
ADVANCES IN THERMODIFFUSION AND THERMOPHORESIS (SORET EFFECT) IN LIQUID MIXTURES
}

\author{
Morteza Eslamian* \\ School of Engineering and Computing Sciences, Texas A\&M University-Corpus Christi, TX, 78412 USA
}

\begin{abstract}
Recent advances in thermodiffusion (Soret effect) in binary and higher multicomponent liquid mixtures are reviewed. The mixtures studied include the hydrocarbon, associating, molten metal and semiconductor, polymer, and DNA mixtures. The emphasis is placed on the theoretical works, particularly models based on the nonequilibrium thermodynamics, although other approaches such as the statistical, kinetic and hydrodynamic approaches are discussed as well. For each mixture, the major theoretical and experimental works are discussed and the research trends and challenges are addressed. Some of the challenges include a need for combining various methods to develop a comprehensive theoretical model or at least to try to understand the differences and pros and cons of each approach. Thermodiffusion and ordinary diffusion coefficients are scarce in some mixtures such as binary DNA, molten metal and metal-semiconductor mixtures and all ternary mixtures. Understanding of the molecular structure of associating mixtures, and modeling of thermodiffusion in such mixtures are also very challenging.

Keywords: Thermal diffusion, Nonequilibrium thermodynamics, Hydrocarbon mixtures, Associating mixtures, Molten metal alloys, Metalsemiconductor mixtures, Polymer mixtures, DNA mixtures
\end{abstract}

\section{INTRODUCTION}

In isothermal mixtures of two or more species (gases, liquids and even solids), mass diffusion occurs if the species are initially distributed unevenly, i.e., when a concentration gradient exists. A temperature gradient can also work as a driving force for mass diffusion, in a phenomenon which is called thermodiffusion, thermal diffusion, or in liquid mixtures Soret (Ludwig-Soret) effect. In thermodiffusion, a time-independent temperature gradient (thermal field) is constantly maintained across a multicomponent mixture, causing all species to become activated and move. Once a thermal field switches on, it immediately exerts thermal forces on all molecules in the mixture, such as the micromolecules and molecules, particulates, ions, and electron, such that all particles are forced to move in the direction of the heat flow, viz. from the hot side to the cold side. Determination of the thermal force is a complex matter; nevertheless, it can be said that the thermal force is proportional to the thermodynamic force in phenomenological equations, which is due to a temperature gradient $\nabla T$. In response to a gradual and continuous migration of all particles to the cold side, a concentration gradient starts to develop within the mixture, which slows down the migration of the initial species to the cold side and exerts counterbalance forces on some of the particles in the opposite direction. The gradually increasing concentration forces then counteract the thermal forces and cause the rearrangement of the species. In the final steady state, all thermal $(\sim \nabla T)$, concentration $(\sim \nabla c)$ and other forces such as electronic forces in ionic mixtures balance one another such that all species remain stationary and motionless. Therefore, in steady state, in a binary mixture, one species is concentrated on the cold side, whereas the other is concentrated on the hot side resulting in the partial separation of species (Eslamian et al., 2010a).
Thermodiffusion has several industrial applications such as the optimum oil recovery from hydrocarbon reservoirs, fabrication of semiconductor devices in molten metal and semiconductor mixtures, separation of species such as polymers, manipulation of macromolecules such as DNA, etc. It is also realized in the atmospheric and oceanic streams at the bottom of the oceans. It is been argued that the life might have originated at the bottom of the oceans due to the replication of DNA molecules as a result of the temperature and $\mathrm{pH}$ gradients (Braun and Libchaber, 2004). Thermodiffusion is also a very interesting theoretical problem. It is a fundamental chemicophysical, hydrodynamics and nonequilibrium thermodynamics problem and as correctly stated by the experts in the field, such as Harstad (2009) and others, it still lacks a clear theory and explanation. In fact, it may be even unrealistic to expect a single theory to predict thermodiffusion in all mixtures from hydrocarbon to associating mixtures as the response of species to a temperature gradient strongly depends on the mixture molecular structure, and the type and characteristics of the mixture, a fact that makes the experimentation and particularly the modeling of thermodiffusion really challenging. It is challenging to devise models that can adequately capture all possible aspects and forces in a mixture that influence the separation of species. In this paper some of the advances in the major branches of thermodiffusion such as hydrocarbons, molten metals and semiconductors, polymers and DNA mixtures will be reviewed and some of the challenges, to our knowledge will be addressed.

\section{THEORETICAL BACKGROUND}

Thermodiffusion was first experimentally observed by Ludwig in the $19^{\text {th }}$ century in a salt liquid solution. Theoretically, however, thermodiffusion was not predicted until sometime after the development of the kinetic theory in the $20^{\text {th }}$ century. Thermodiffusion

*Phone: 1-361-825-3652; Email: Morteza.Eslamian@tamucc.edu 
is a nonequilibrium and second order effect, which did not appear in the equations when the kinetic theory of gases was first developed for the rarefied gases. Rarefied gases are governed by the Maxwellian velocity distribution, and for this distribution, thermodiffusion coefficient is predicted to be zero. This was first recognized by Enskog and independently and at about the same time by Chapman who obtained a similar result. The Chapman-Enskog method of finding the thermodiffusion coefficients proceeds through solving the Boltzmann equation with a perturbed Maxwellian velocity distribution (the nonequilibrium case) and presents the phenomenological coefficients in terms of collision integrals, which require substantial mathematical and numerical procedures. Recently, Debbasch and Rivet (2010), using the Boltzmann equation, have proposed stochastic models and description of thermodiffusion in dilute gases. Their findings show that a temperature gradient induces contributions to the friction coefficient, acting on the diffusion particles. Although thermodiffusion theory in gases was first realized based on the kinetic theory, nonequilibrium thermodynamics is equally applicable to both gases and liquids.

Thermodiffusion even in systems as simple as the binary linear hydrocarbon mixtures is still a complex phenomenon. It is a kinetic or nonequilibrium problem and at the same time, a low velocity hydrodynamics problem as well. As the mixture molecular structure and the interaction between molecules become more complex, such as in associating mixtures, chemical and molecular physics start to play an important role as well. Molecular dynamics simulations and also experimental observations show that usually a higher molecular mass, smaller diameter, higher density and higher moment of inertia cause a species to segregate on the cold side. Besides, the intermolecular or chemical effects among like and unlike molecules play a major role. This highly multidisciplinary trait of thermodiffusion has sometimes resulted in the lack of effective connection and communication among engineers, physicists and chemists working on this area. Due to the complexity of the problem, it is hard for the researchers who work on thermodiffusion to master all aspects of the problem. There is also not enough collaboration among the researchers in the field.

This author believes that at least for simple mixtures by far the most successful and widely used approach used in thermodiffusion modeling is the nonequilibrium thermodynamics, exemplified by the work of de Groot and Mazur (1984), Prigogine et al. (1950), and Drickamer and coworkers (1955a 1955b, 1956) who have had perhaps the most contribution in the establishment of the theory in the past. The major setback of the nonequilibrium thermodynamics formulation that hampers satisfactory advancement of the theory is the appearance of a quantity called the neat heat of transport. The dependence of the thermodiffusion factor on the net heat of transport may reflect the inadequacy of the nonequilibrium thermodynamics to completely model thermodiffusion, as thermodiffusion depends on the hydrodynamics of the mixture as well as its thermodynamics.

There are several models that are built upon various approaches of the hydrodynamics theory such as those of Semenov and Schimpf (2005) and Brenner and Bielenberg (2005). Employment of the kinetic theory of liquids and stochastic methods is another approach, e.g., (Shapiro 2004; Bringuier 2011). The drawback of such models is their dependence on one or more unknown or undetermined parameters and/or their limited applicability to some simple cases such as dilute solutions, which makes them difficult to be practically examined against the experimental data. Unfortunately, there has been no or little connection between these different approaches. To summarize, one may roughly categorize the thermodiffusion models according to the characteristics outlined in section 2.1.

\subsection{Major modeling approaches}

\section{Dynamic or kinetic models vs. static models}

Based on this measure, those models that consider the transient and kinetic nature of thermodiffusion are called dynamic or kinetic models, and those that merely rely on thermostatic properties of the mixture are called static models. In this context, the Kempers $(1989,2001)$ and the Haase (1969) models and to some extent the Firoozabadi et al. (1998, 2000) models that rely on the enthalpy or internal energy of the mixture to estimate thermodiffusion coefficients are static models, whereas the Drickamer et al. (1956) model, and its modified version, the EslamianSaghir model expression (2009a) that incorporate the activation energy of viscous flow, instead of molar enthalpies or internal energies of the species, are examples of dynamic models. All of the mentioned models are within the framework of thermodynamics. Also, obviously statistical models based on the kinetic theory, such as the Bringuier (2011) model are kinetic or dynamic as well.

It's been shown that the prediction ability of the Kempers model (a static model) is acceptable for high pressure mixtures in which case the mobility of the system has declined due to the high pressure of the system. In complex mixtures at near atmospheric pressures, a static model cannot adequately capture all complexities of the problem, particularly for mixtures with a complex molecular structure. The Kempers expression has been derived using the classical or equilibrium thermodynamics approach. The Haase expression for thermodiffusion, however, is just a speculation (later derived by Kempers using classical thermodynamics). Haase (1969) derived an expression for the pressure diffusion factor of binary liquid mixtures in a thermally equilibrium system and then extended it to the thermodiffusion problem as well. Obviously, the kinetic nature of thermodiffusion is not considered in the Haase expression for thermodiffusion factor. On account of the kinetic effects, Kempers in his second paper (2011) incorporates a matching parameter, whose value is obtained by comparison of the model predictions with the experimental data. As pointed out by Bringuier (2011), pressure diffusion is an equilibrium or static effect, whereas thermodiffusion is a non-equilibrium and kinetic effect. Therefore, even though some simple models such as the Kempers and Haase may work for some simple mixtures at high pressures and close to the critical point, a theoretically sound thermodiffusion model must be dynamic, kinetic and nonequilibrium, in order to be able to predict the thermodiffusion coefficients in wide range of mixtures.

\section{Matching-parameter vs. stand-alone models}

Most thermodiffusion models involve several parameters one or some of which are unknown or undetermined, and this is due to the statistical and nonequilibrium nature of the thermodiffusion problem. Unfortunately, this complexity hampers the practicability and prediction ability of such models. In fact having one or more matching parameters is inevitable in many cases when a mixture with a complex molecular structure is involved. Perhaps half of the effort in the development of practical expressions for the estimation of thermodiffusion coefficients is to find a way to estimate the matching or undetermined parameters. Some workers have come up with approximate values for the unknown parameters whereas in many other cases, the unknown parameters are used as a matching parameter with inadequate physical justification behind them. Static models such as the Haase model are usually stand-alone models. Drickamer et al. (1956) and Eslamian-Saghir (2009a) expressions are based on nonequilibrium thermodynamics where the net heat of transport is simulated and replaced by the activation energy of viscous flow turning the models to dynamic and kinetic, yet stand-alone models.

\section{Hydrodynamics, nonequilibrium thermodynamics, and kinetic theory models}

In hydrodynamic models, usually applied to thermophoresis of large particles in a solvent, the solvent is treated as a continuum; the formulation starts with writing the momentum equation for the large molecules flowing in the solvent, considering the pressure and velocity terms; the challenge is to model the local pressure gradients and force fields around the molecules or macromolecules in the mixture. 
In nonequilibrium thermodynamics approach, the formulation starts from the entropy production rate in a multicomponent mixture assuming that local equilibrium pertains in the elements of the fluid. The entropy production rate is expressed in terms of heat and mass fluxes and finally the fluxes are expressed as linear functions of thermodynamics driving forces, i.e. temperature and chemical potential gradients. In the nonequilibrium thermodynamics approach, a quantity arises, which is called the enthalpy or heat of transport, which has to be determined from outside of the nonequilibrium thermodynamics, perhaps from hydrodynamics or kinetics of the problem. The challenge begins here, as the heat of transport strongly depends on the type and molecular structure of the mixture.

Bringuier (2011) uses a statistical mechanics approach in the framework of the kinetic theory of liquids. The final expression involves a collision integral which is a function of the mean free paths and particle momenta. The kinetic approach of Bringuier is a robust mathematical approach, although it relies on some assumptions for instance the elastic collision of the particles, or in the case of the Kincaid et al. (1987) work, the hard sphere assumption. Some other workers including Drickamer et al. (1956), Firoozabadi et al. (1998, 2000) and the present author et al. (2009a), among others, have used a combined theoretical and empirical approach. This approach is based on the Eyring's rate theory and the concept of the activation energy of viscous flow. Thermodiffusion is an activated process in that species are activated and move as a result of a temperature gradient. Drickamer tried to link thermodiffusion with the fluid flow in a viscous flow, in a systematic manner. The essence of his modeling approach is based on the assumption that the net heat of transport has a close relationship with the activation energy of viscous flow, the energy required to put the unit of the fluid in motion. Note that since the activation energy of viscous flow is temperature dependent, this modeling approach renders a practicable dynamic expression for the thermodiffusion factor. In contrast, the kinetic theory approach is more robust and mathematically sound and may provide a physical description, but it still fails to provide a ready-to-use expression for the estimation of the thermodiffusion coefficients.

In the hydrodynamic approach, usually applied to thermodiffusion of large molecules in a solvent, often called thermophoresis, the NavierStokes or the momentum equation is written to describe the motion of large molecules in a solvent, e.g., (Semenov and Schimpf, 2005). The challenge is to simulate the force and pressure terms acting on the large molecules, induced as a result of the imposed temperature gradient. Note that in the nonequilibrium thermodynamics approach, for instance, the pressure gradient is assumed to be locally and globally zero within the mixture and around the molecules. This approach, similar to the kinetic theory of liquids, demands some mathematical work. The expression proposed for the Soret coefficient by Semenov and Schimpf (2005) contains several undetermined matching and modeling parameters rendering the expression impractical, at least for now. Alternatively, Brenner (2006) has proposed a simplified hydrodynamic/Brownian approach for thermophoresis of "inert" large molecules in dilute solutions, later extended to non-dilute and also gas mixtures. In his model expression, the thermodiffusion coefficient is expressed as a function of the solvent's self-diffusivity and thermal expansivity as well as a matching parameter of order 1 . The undetermined matching parameter has been incorporated to the model on account of the chemical effects between the solute and solvent molecules; the chemical effects may even cause a sign-change in the thermodiffusion coefficient with a change in the molecular weight and temperature. In fact, the main challenge in thermodiffusion modeling is to develop models that can, to some extent, account for the chemical effects and complex interfacial forces.

\section{Other approaches}

In addition to the classical or conventional approaches, i.e., equilibrium and nonequilibrium thermodynamics and hydrodynamics methodologies discussed above, there are a number of other less- conventional or hybrid approaches as well. For instance, Morozov (2009) in an attempt to model thermodiffusion in molecular mixtures, uses a statistical mechanics approach originally developed by Bearman and Kirkwood (1958). In that approach, a non-zero local pressure gradient is assumed around the molecules, similar to the hydrodynamics approach commonly used in thermophoresis. He revisited and corrected the Bearman and Kirkwood formulation for the partial pressure of each component in a binary mixture. He assumes that the driving force of thermodiffusion has an equilibrium part and a nonequilibrium part. As it can be expected, the equilibrium term is easier to formulate, whereas similar to other approaches, the nonequilibrium term, which may be negligible in simple mixtures but profound in many other complex mixtures, does not have a general form and depends on the molecular structure of the mixture. The developed expression encompassing only the equilibrium forces, still requires several parameters and properties of the components such as the compressibility factors to be determined. In order to estimate the undetermined parameters, he uses a perturbation theory assuming that the species behave as hard-spheres. Some of these unknown parameters could be obtained from an equation of state, perhaps. He compares his results against benzene-cyclohexane mixture, which is considered as a complex mixture among hydrocarbon mixtures owing to the occurrence of a sign change when concentration changes, where a fair agreement is observed including the prediction of the sign change. We note that only very few models can predict a sign change in benzene-cyclohexane mixture, e.g., (Eslamian and Saghir, 2012a). Debuschewitz and Kohler (2001) identified two effects in benzene-cyclohexane mixture, one called the isotope effect stemming from the difference in mass and moments of inertial of the two species and a less-known chemical effect, which is strongly composition dependent and is due to the intermolecular forces, which change with the molecular structure of the mixture.

Recently, Semenov and Schimpf (2009) used the idea of variable local pressure (hydrodynamics approach) but within the framework of the linear nonequilibrium thermodynamics. This is in fact an attempt to combine nonequilibrium thermodynamics with hydrodynamics. They expressed chemical potential in terms of the mixture number density, temperature and the local pressure. As mentioned before, the common practice in nonequilibrium thermodynamics is to assume a constant pressure everywhere within the mixture such that $\nabla P=0$. Alternatively, to maintain a mechanical equilibrium in the mixture, the local pressure gradient may be expressed in terms of the number density and temperature gradients (Gibbs-Duhem expression). The local mechanical equilibrium indicates that the forces acting on a small element of the fluid in a closed system, originate from the number density, temperature and pressure differences across an element. The net force acting on the molecules causes mass diffusion within the mixture. Once gradually a force balance on moving segments is established, the system reaches a stationary or steady state. Using the variable pressure approach, Semenov and Schimpf expressed the mass fluxes in terms of temperature and volume fractions. They noticed a discrepancy in their equations and in order to resolve the problem, concluded that the heat of transport of each component has to be equal to the chemical potential of the same component. This conclusion is problematic as the heat of transport is a dynamic or nonequilibrium property of the mixture whereas the chemical potential is a thermostatic property, i.e. it is associated with an equilibrium state. Overall the issue of the local pressure and its variation has not been understood well and further research is needed to physically understand and mathematically model the pressure change in the mixture and its relationship to thermodiffusion.

Recently, Semenov and Schimpf (2011) used a thermodynamic approach supplemented by statistical mechanical calculations and expanded and elaborated the work of Semenov (2010). They developed an expression for the Soret coefficient for dilute mixtures that clearly shows the intermolecular or chemical effects, the mass difference effects and moment of inertia difference effect. Their expression is 
mostly dependent on the chemical potentials and some undetermined parameters such as the energy of interaction, etc.

There are some other models as well as those described above: the statistical model of Prager and Eyring (1953), radiation pressure model of Gaeta (1969), and fluctuation model of Shapiro (2004), among others. The recent theory of Shapiro is based on the statistical theory of fluctuations around an equilibrium state. The Onsager matrix of phenomenological coefficients is expressed in terms of the penetration lengths. Despite its robust mathematical approach, its drawback is its dependence on several undetermined parameters.

Kocherginsky (2010) suggests an interest and compact approach to describe Soret, Dufour and Seebeck effect. The description is based on the idea that the rate of mass transport is proportional to the gradient of physicochemical potential (rather than only chemical potential for instance), which is a general function and includes the product of molar entropy and temperature. As a result, an expression is obtained for the Soret coefficient which is a function of molar entropy (and also concentration and temperature), rather than the net heat of transport:

$S_{T}=\left(\ln c-\frac{\bar{s}}{R}\right) \frac{1}{T}$

where $S_{T}$ is the Soret coefficient, $c$ is the mixture concentration, $R$ is the universal gas constant, $\bar{s}$ is the molar entropy, and $T$ is the mixture average temperature. The above expression can theoretically predict a sign change. The expression is quite simple and neat; however, the evaluation of the partial molar entropy in a nonequilibrium mixture is as difficult as modeling of the net heat of transport in nonequilibrium thermodynamics models. It is not clear to us that whether the molar entropy can be obtained from an equation of state or not. Moreover, it seems that similar to the Kempers and Haase models, which are functions of thermostatic properties such as molar enthalpy and molar volume, Eq. (1) is also a function of the molar entropy, a thermostatic property and therefore can be only valid for mixtures not too far away from equilibrium.

\section{Review papers}

There are several review papers that consider various aspects of thermodiffusion modeling. These include but are not limited to the following: Faissat et al. (1994) who discuss dynamic versus static models. They focus on the thermodynamics and nonequilibrium thermodynamics modeling approaches with emphasis on thermodiffusion in porous media (hydrocarbon reservoirs). They provide some insight on the mechanism of thermodiffusion on the molecular level. Gonzales-Bagnoli et al. (2003) used the equilibrium and nonequilibrium thermodynamics models of Drickamer et al. (1956), Kempers (2001), Haase (1969), and Shukla-Firoozabadi (1998) and used them to evaluate thermodiffusion factors in various hydrocarbon mixtures.

Harstad (2009) provide a review on the thermodiffusion modeling in the context of nonequilibrium thermodynamics and provide useful discussions on various models and a description of the process in the molecular level. Issues such as the concept of the heat of transport are discussed as well as the significance and progress in the area of the molecular dynamics simulations. He also argues that due to the importance of the intermolecular and interfacial forces and their impact on the overall behavior of thermodiffusion, it is hard to imagine one single thermodiffusion model can adequately capture the effect in all phases. We further wish to add that even it is hard to imagine that a model can be used equally for two different types of liquid mixtures, for instance for hydrocarbons and molten metal mixtures.

Farago et al. (2006) using a variational approach obtained an equation for the thermodiffusion factor as a function of a variable that plays the role of the net heat of transport. If this function is replaced by a dynamic property such as the net heat of transport, the nonequilibrium thermodynamics formula to be discussed later, Eq. (2) is obtained, whereas if it is replaced by the partial enthalpy, the Haase and Kempers equations are obtained. The choice of the function depends on our interpretation of the thermodiffusion problem and the constraint we apply on the problem. Farago et al. argue that the Kempers constraint on a flat pressure across the mixture results in the Kempers expression, which is not quite correct.

Demirel and Sandler (2001) provide a rather clear description of coupled heat and heat transfer phenomena including the thermodiffusion theory. Issues such as the phenomenological equations and coefficients, frame of reference, and heat of transport are described.

Eslamian and Saghir (2009b) reviewed some of the thermodiffusion models. Their emphasis was placed on the meaning and the method of calculation of the activation energy of viscous flow introduced by Eyring and coworkers (Glasstone et al., 1941).

Wiegand (2004) reviewed the thermodiffusion and thermodiffusion in liquid solutions, with an emphasis on the working principles of some of the experimental methods, molecular dynamics simulations, thermodiffusion in polymer solutions, colloids, and thermodiffusion near the critical point.

Major experimental methods used to obtain thermodiffusion coefficients have been briefly reviewed in a recent work by Srinivasan and Saghir (2011) and in an earlier work by Platten (2006). Platten also reviews some experimental works on hydrocarbon mixtures with an emphasis on the importance of having reliable benchmark databases for model validation. Some of the experimental methods include but are not limited to: elementary Soret cell method, beam deflection technique, thermal diffusion forced Rayleigh scattering technique, and the thermogravitational column method.

\subsection{Velocity frame of reference}

Here we demonstrate the significance and role of the frame of reference in the measurements and prediction of thermodiffusion coefficients. In many research works, theoretical or experimental, diffusion coefficients (thermal or ordinary) are measured and presented without specifying a frame of reference. This implies that the fluxes are measured with respect to a fixed point or a fixed frame, a reference frame which is called the laboratory frame of reference. Using a fixed frame, however, can be problematic and erroneous, for instance if the volume of the experimental cell changes. To suppress this problem, it is advisable to express the measurements with respect to a mean-velocity such as the velocity of the center of mass, center of volume, etc. Note that if the change in the volume of the liquid in the experimental cell is negligible, the center of volume of the cell will remain stationary during the experiments, in which case the fixed or laboratory frame of reference coincides with the volume frame of reference. This is usually the case in most thermodiffusion experiments in liquids, i.e. the experimental data are expressed tacitly in a volume frame of reference.

In a multicomponent system of $n$ species in various frames of reference, the maximum number of independent ordinary diffusion coefficients is $(n-1)^{2}$ (Brady, 1975). As a result, for a binary mixture there is only one binary diffusion coefficient (and also one thermodiffusion coefficient), which is unique and independent of the frame of reference. Therefore in a binary mixture, diffusion coefficients expressed in a volume frame of reference are identical with those expressed in any other frame of reference. In ternary and higher mixtures, however, the diffusion and thermodiffusion coefficient are frame of reference dependent. In a binary liquid or gas mixture (and tentatively any multicomponent mixture), the Soret coefficient and thermodiffusion factor are independent of the frame of reference and therefore they are the best data to report by the experimentalists (Eslamian et al., 2012b).

It is interesting to note that despite the fact that in binary mixtures, thermodiffusion coefficients are independent of the frame of reference, some models propose expressions in various frames of reference. For instance, the Kempers model, the Haase model and the Drickamer model. Besides, the definition of the frame of reference in the Kempers model is erroneous. The bottom line is that in a binary mixture, any 
model proposing multiple thermodiffusion coefficients or factors for various frames of reference is fundamentally wrong, even though it may be successful in some cases. Bringuier (2011) comments on a similar issue as well.

\section{HYDROCARBON MIXTURES}

Thermodiffusion has been widely studied in the hydrocarbon mixtures more than any other mixture, e.g., (Blanco et. al, 2007 and 2010; Van Vaerenbergh, 2009; Eslamian et. al, 2010b; Eslamian and Saghir, 2009a; Shukla and Firoozabadi, 1998). This is due to the absence or weak appearance of the chemical effects in linear and nonpolar hydrocarbon mixtures and of course due to the presence of thermodiffusion in non-isothermal oil reservoirs. Due to the porous nature of underground oil reservoirs, a few studies have been performed on the reservoir composition under the influence of a temperature gradient, e.g., (Faissat et al., 1994; Colombani et al., 2002; Jaber et al., 2005). The oil industry is in particular interested in this kind of research, owing to the role of thermodiffusion in determining the accurate composition of underground reservoirs for optimum oil recovery. In fact, some of the key research works and publications on thermodiffusion have been produced in the oil industry, e.g., the Kempers and Firoozabadi et al. models. In hydrocarbon mixtures usually there is no sign change with a change in the mixture composition and temperature (Eslamian and Saghir, 2009a). This is because the molecular structure of the hydrocarbon mixtures only slightly changes with the mixture composition and temperature variation.

\subsection{Experimental studies on hydrocarbon mixture}

Experimental data are rather abundant in various combinations of binary hydrocarbon mixtures, e.g., (Perronace et al., 2002; Polyakov et al., 2006, 2009; Leahy-Dios et al. 2008). In some particular cases, benchmark experimental data are available obtained using very different methods with minimum uncertainty (Platten, 2006). There are also few sets of hydrocarbon experimental data on ternary and quaternary mixtures some of which performed onboard the International Space Station (Van Vaerenbergh et al., 2009; Leahy-Dios, 2005; Königer et al., 2010; Blanco et al., 2010).

The experimental data, either binary or ternary have received enough attention in other publications. One ongoing and controversial area of research is to study the effect of vibration (g-jitter) on the accuracy of the experimental data taken during space missions (Yan et al., 2005; Shevtsova et al., 2007; Srinivasan et al., 2010, Shevtsova et al., 2011). It is not quite clear how and to what extent the micro-vibrations induce remixing of species and deteriorate the thermodiffusion experimental data. Space experiments are obviously very expensive to perform and therefore it is important to ensure that the resources in the fluids dynamics laboratory onboard the international space station are spent wisely.

\subsection{Theoretical studies on hydrocarbon mixtures}

For hydrocarbon mixtures, the classical approach of the nonequilibrium thermodynamics combined with some version of the Eyring's activation energy of viscous flow provides the best performance with minimum effort. Following the phenomenological equations, it can be shown that, the thermodiffusion factor, Error! Objects cannot be created from editing field codes.of the first component is obtained as follows, e.g., (see Eslamian et al., 2010b for a detailed derivation):

$\alpha=\frac{Q_{1}^{*}-Q_{2}^{*}}{x_{1}\left(\partial \mu_{1} / \partial x_{1}\right)}$

where $Q_{k}^{*}$ is the net heat of transport of component $k, x_{I}$ is the mole fraction of component 1, and Error! Objects cannot be created from editing field codes.is the chemical potential of component $1 . Q_{k}^{*}$ is mathematically defined as follows (Demirel and Sandler, 2001):

$Q_{k}^{*}=\left(\frac{\left(\mathbf{j}_{q}^{\prime}\right)_{k}}{\mathbf{j}_{k}}\right)_{T} \quad$ at $\quad \nabla T=0$

where $\left(\mathbf{j}_{q}^{\prime}\right)_{k}$ is the pure heat flux and $\mathbf{j}_{k}$ is the mass flux, associated with component $k$. Eq. (3) indicates that the net heat of transport of component $k, Q_{k}^{*}$ is the conductive heat flow of the diffusing component $k$, per particle, mole or kilogram, depending on the unit mass adopted, required to be absorbed by the local region to keep the temperature of the region constant. $Q_{k}^{*}$ can also be expressed in terms of the phenomenological coefficients (de Groot and Mazur, 1984).

Dougherty, Jr. and Drickamer (1955a and 1955b) and Tichacek et al. (1956) provide other versions of Eq. (2) as well, in an attempt to estimate thermodiffusion in various frames of reference. However, after the discussion of section 2.2 , it is clear that at least in a binary mixture, there is only one thermodiffusion factor regardless of the frame of reference. Therefore, other versions of Eq. (2), incorporating for instance, molar weights or volumes are erroneous.

Under the influence of a temperature gradient, structural units of the mixture, such as molecules, atoms or clusters need some energy to overcome the bonds and free themselves from their neighbors and jump to the next available site in the mixture. The required energy for this transition to occur may be regarded as the net heat of transport $Q_{k}^{*}$. Although not identical, flowing of a viscous fluid may be considered as a process similar to mass transfer in thermodiffusion in that in both phenomena some sort of activation energy is required so as to overcome the cohesive energy between molecules and set them in motion. Drickamer and coworkers, among others, have systematically studied the possibilities for simulating the net heat of transport with some sort of the activation energy of viscous flow. Eslamian and Saghir (2009a) showed that it is more justified to assume that the net heat of transfer of each component equals the activation energy of viscous flow of the same component in the mixture. However, since the activation energy of viscous flow for a component in a mixture is not defined, the activation energy of viscous flow of a pure component may be considered as the net heat of transport of that component in the mixture $\left(Q_{i}^{*}=E_{i}^{v i s}\right)$. Thus, in a simple binary mixture, Eq. (2) takes the following form:

$\alpha=\frac{E_{1}^{v i s}-E_{2}^{v i s}}{x_{1}\left(\partial \mu_{1} / \partial x_{1}\right)}$

Other model expressions that have been widely used to estimate thermodiffusion factor in hydrocarbon mixtures include but are not limited to the Haase, Kempers and Sukla-Firooazabadi expressions. It was argued before that the Haase and Kempers models are erroneous or at least only applicable to near-equilibrium and high pressure conditions, as they are static models. The Haase and Kempers formulas for binary mixtures are written as follows:

$$
\begin{aligned}
\text { Haase (1969): } & \alpha=\frac{M_{1} \bar{H}_{2}-M_{2} \bar{H}_{1}}{\left(M_{1} x_{1}+M_{2} x_{2}\right) x_{1}\left(\partial \mu_{1} / \partial x_{1}\right)} \\
\text { Kempers (2001): } & \alpha=\frac{\bar{V}_{1} \bar{H}_{2}-\overline{V_{2}} \bar{H}_{1}}{\left(\bar{V}_{1} x_{1}+\bar{V}_{2} x_{2}\right) x_{1}\left(\partial \mu_{1} / \partial x_{1}\right)}+\alpha_{0}
\end{aligned}
$$

where $\bar{H}_{k}$ is the molar enthalpy, $\bar{V}_{k}$ is the molar volume, and $M_{k}$ is the molar weight of component $k$. Note that Kempers derived Eq. (5), using 
an equilibrium thermodynamics approach, whereas Haase, only guessed Eq. (5), by comparing thermodiffusion with pressure diffusion. $\alpha_{0}$ in

Eq. (6) is an undetermined matching parameter introduced in the 2001 version of Kempers model, on account of the kinetic effects.

Based on the work of Dougherty Jr. and Drickamer (1955a, 1955b), Shukla and Firoozabadi (1998) modeled the net heat transport for component $i$ in the mixture as a function of the thermostatic properties of the components, particularly the internal energy:

Shukla-Firoozabadi (1998)

$$
\alpha=\frac{\overline{V_{1}} \bar{V}_{2}}{\bar{V} x_{1}\left(\partial \mu_{1} / \partial x_{1}\right)}\left[\frac{\overline{U_{1}}}{\tau \overline{V_{1}}}-\frac{\overline{U_{2}}}{\tau \bar{V}_{2}}\right]
$$

where $\bar{U}_{k}$ is the molar internal energy of component $k$. Note that the parameter $\tau$ in Eq. (7) takes a constant value of 4 in their model; it is the ratio of the vaporization energy of a liquid to its activation energy of viscous flow and is not a constant in general, but its value is close to 4 for most linear hydrocarbons. This constant 4 had been proposed by Drickamer and coworkers. The Shukla-Firoozabadi model in the form of Eq. (7) is in fact a static expression and therefore, to make it a dynamic or kinetic model, a variable has to be used instead of constant 4. It has been shown that using a variable ratio of vaporization energy to activation energy generates better estimates of the thermodiffusion factors, e.g., (Yan et al. 2008).

To improve the prediction power of the Firoozabadi model, Abbasi et al., (2011) used the concept of free volume to estimate the ratio of the evaporation energy to the activation energy of viscous flow $\tau$, appearing in Eq. (7). Based on this concept, free volume controls the diffusivity of the molecules in diffusion-limited systems. It states that the transfer kinetics of molecules depends greatly on molecular size and shape as well as the concentration.

Figure 1 shows experimental data (Leahy-Dios et al., 2008) of thermodiffusion coefficients, $D_{T}$, of $n \mathrm{C}_{10}-n \mathrm{C}_{i}$ alkane mixtures in room temperature and atmospheric pressure, versus the number of carbon atoms in $\mathrm{C}_{i}$ component. The prediction power of three expressions, viz., Eqs. (4), (5), and (7) are also displayed, (Eslamian and Saghir, 2009a). The data shown in Figure 1 do not necessarily indicate that the same trend will occur for other mixtures, as well. Nevertheless, we note that the Haase expression (and also Kmepers) fail to predict even the sign of the thermodiffusion coefficient. At higher pressures, however, the Haase and Kempers model expressions work fairly well. Figure 2 shows the experimental data (Rutherford and Roof, 1959) and the prediction of the Haase expression, Eq. (5), for the thermodiffusion factor $\alpha$ of methane- $n$-butane mixture versus pressure (Eslamian and Saghir, 2009a).

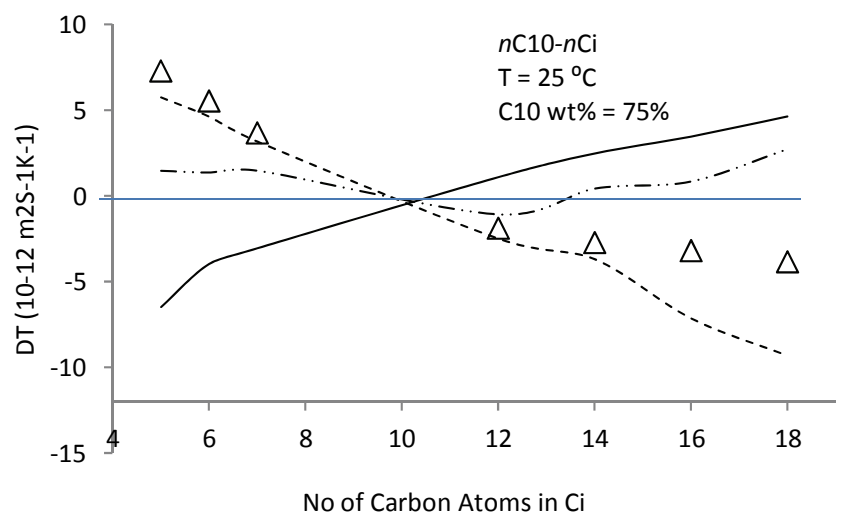

$$
\begin{aligned}
& \Delta \text { Expt. Data } \quad-\cdots-\cdots \text { Shukla-Firoozabadi } \\
& \text { - Haase-Kempers (Mass) _. - - - Eslamian-Saghir }
\end{aligned}
$$

Fig. 1 Variation of the thermodiffusion coefficient $D_{T}$ of $n \mathrm{C}_{10}-n \mathrm{C}_{i}$ alkane mixture versus the number of carbon atoms in $\mathrm{C}_{i}$.
(Eslamian and Saghir, 2009a; expt. data from Leahy-Dios et al., 2008).

To our knowledge, there are only three major models that provide expressions for the thermodiffusion coefficients in mixtures with more than two components, i.e., ternary, quaternary, etc. These models are the Kempers (2001), the Firoozabadi et al., (2000) and Eslamian et al. expressions $(2010 \mathrm{~b}, 2010 \mathrm{c})$. We have already substantiated that the Kempers model is not reliable due to the lack of the clarity on the contribution of the kinetic effects and also confusion on the role of the velocity of reference.

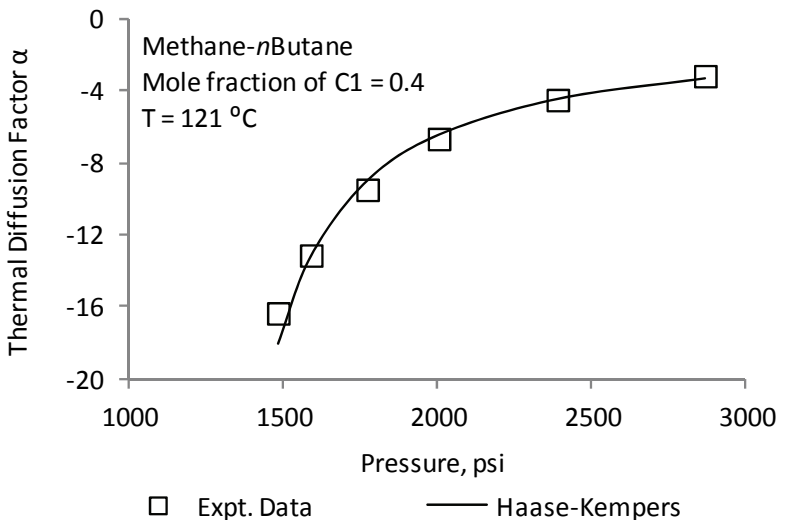

(a)

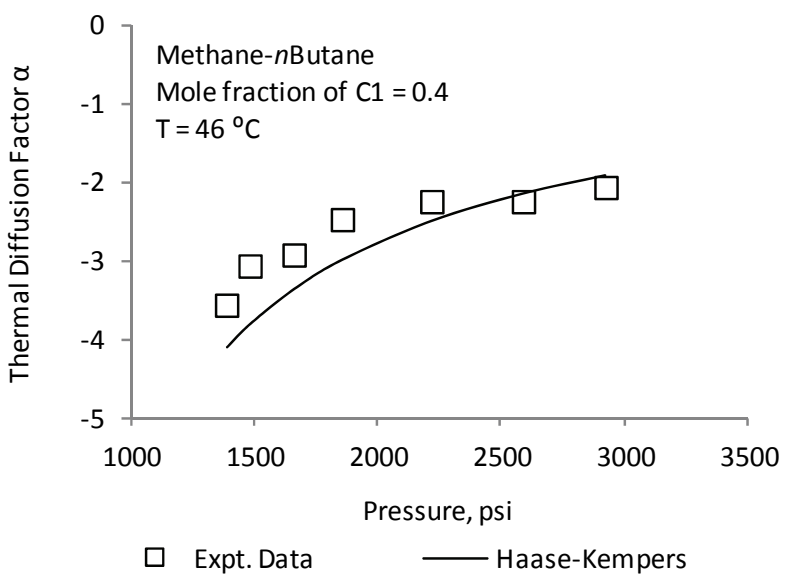

(b)

Fig. 2 Variation of the thermodiffusion factor $\alpha$ of methane- $n$-butane versus pressure; (a) at $121{ }^{\circ} \mathrm{C}$ and (b) at $46{ }^{\circ} \mathrm{C}$. (Eslamian and Saghir, 2009a; expt. data from Rutherford and Roof, 1959).

In the Firoozabadi multicomponent model (Firoozabadi et al., 2000), the phenomenological coefficients (PC), $L_{i j}$, are correlated with the ordinary diffusion coefficients; PCs have to be calculated before the thermodiffusion coefficients can be estimated. The mole-based thermodiffusion coefficients $\widetilde{D}_{T i}^{x}$ where $\left(\sum \widetilde{D}_{T i}^{x}=0\right)$ are expressed in terms of the phenomenological coefficients and the net heats of transport as follows:

$\tilde{D}_{T 1}^{x}=\frac{1}{c T^{2}}\left(L_{11} \frac{Q_{1}^{*}}{M_{1}}+L_{12} \frac{Q_{2}^{*}}{M_{2}}-\left(L_{11}+L_{12}\right) \frac{Q_{3}^{*}}{M_{3}}\right)$

$\widetilde{D}_{T 2}^{x}=\frac{1}{c T^{2}}\left(L_{21} \frac{Q_{1}^{*}}{M_{1}}+L_{22} \frac{Q_{2}^{*}}{M_{2}}-\left(L_{21}+L_{22}\right) \frac{Q_{3}^{*}}{M_{3}}\right)$ 
We note that while in the Shukla-Firoozabadi binary model (Eq. 7), the thermodiffusion factor is independent of the phenomenological coefficients, in the extension of the model to multicomponent mixtures, the thermodiffusion factors become dependent on the phenomenological coefficients. Careful inspection of the FiroozabadiGhorayeb-Shukla multicomponent model reveals an ambiguity in their derivation: they never use the condition of steady state in which all mass fluxes are zero. In their derivation using Eqs. (10) and (11) of Firoozabadi et al., (2000), combined with the definition of the thermodiffusion coefficients, one can eliminate $\nabla T$ and $\nabla \mu$ to obtain the thermodiffusion coefficients. This is in fact the approach that the present author and coworkers used for the derivation of their multicomponent formula (Eslamian et al., 2010b and 2010c). Despite this ambiguity in the derivation of the Firoozabadi-Ghorayeb-Shukla model, its prediction power for the multicomponent mixtures is in the correct order of magnitude although the signs of the thermodiffusion coefficients cannot be predicted in some cases (Eslamian et al., 2010b). As mentioned above, the theory of nonequilibrium thermodynamics used to develop Eq. (2) can be followed to obtain expressions for the thermodiffusion coefficients in terms of the net heat of transport in ternary and higher multicomponent mixtures. Using the Eyring's rate theory of viscous flow, similar to Eq. (2) that was converted to Eq. (4), the following practicable expressions have been suggested (Eslamian et al., 2010b, 2010c).

$$
\begin{aligned}
& \widetilde{D}_{1}^{T}=\frac{D\left(E_{1}^{v i s}-E_{3}^{v i s}\right)-B\left(E_{2}^{v i s}-E_{3}^{v i s}\right)}{T(A D-B C)} \\
& \widetilde{D}_{2}^{T}=\frac{-C\left(E_{1}^{v i s}-E_{3}^{v i s}\right)+A\left(E_{2}^{v i s}-E_{3}^{v i s}\right)}{T(A D-B C)}
\end{aligned}
$$

where parameters $A, B, C$ and $D$ are related to the elements of the ordinary diffusion matrix and also the derivatives of chemical potential with respect to concentration. In multicomponent mixtures, the thermodiffusion coefficients are defined in such a way that their sum is zero. The prediction power of both Firoozabadi et al., (2000) and Eslamian et al., models (2010b, 2010c) are comparable, when evaluated against the hydrocarbon mixtures.

Table 1 Thermodiffusion coefficients $\widetilde{D}_{T}^{i} \times 10^{12}\left(\mathrm{~m}^{2} / \mathrm{sK}\right)$ for THN-IBBC10 ternary mixture at $T=298 \mathrm{~K}$ and $P=0.1 \mathrm{MPa}$.

\begin{tabular}{|l|l|l|l|l|}
\hline$\widetilde{D}_{T}^{i} \times 10^{I 2}$ & Expt. data & $A^{a}$ & $B^{b}$ & $C^{c}$ \\
\hline & \multicolumn{4}{|c|}{$(0.333,0.333,0.333) \mathrm{wt} . \%$} \\
\hline THN & 1.36 & 1.40 & 1.99 & 1.08 \\
\hline IBB & 0.24 & 0.25 & -0.68 & -0.52 \\
\hline C10 & -1.57 & -1.64 & -1.31 & -0.57 \\
\hline & \multicolumn{4}{|c|}{$(0.600,0.200,0.200)$ wt. $\%$} \\
\hline THN & 1.34 & 1.34 & 1.99 & 0.93 \\
\hline IBB & -0.16 & -0.15 & -0.78 & -0.48 \\
\hline C10 & -1.18 & -1.18 & -1.21 & -0.44 \\
\hline
\end{tabular}

${ }^{\mathrm{a}}$ Firoozabadi-Ghorayeb-Shukla model (Firoozabadi et al, 2000).

${ }^{\mathrm{b}}$ Kempers Mass-Based model (Kempers, 2001).

${ }^{c}$ Non-equilibrium thermodynamics, (Eslamian et al., 2010b).

Here we display some corrected results on the performance of multicomponent models, first reported by Eslamian et al., (2010b). In that reference, the experimental data of component 1 and 2 had been swapped by error. Experimental data and model predictions are massbased. Mixtures components are 1,2,3,4-tetrahydronaphtalene (THN), isobutylbenzene (IBB), n-dodecane (C12), n-decane (C10), and cis-1,2dimethylcyclohexane $(\mathrm{C} 12 \mathrm{DMCH})$. The ternary experimental data (Tables 1 and 2) are taken from Blanco et al., (2010) and the quaternary experimental data (Table 3) are taken from Van-Vaerenbergh et al., 2009. Results show that for a range of concentrations and mixture type, although the Firoozabadi et al., (2000), and Eslamian et al., (2010b) models are partially successful, still none of the models can adequately predict the multicomponent experimental data.

Table 2 Thermodiffusion coefficients $\widetilde{D}_{T}^{i} \times 10^{12}\left(\mathrm{~m}^{2} / \mathrm{sK}\right)$ for THN-IBBC12 ternary mixture at $T=298 \mathrm{~K}$ and $\mathrm{P}=0.1 \mathrm{MPa}$.

\begin{tabular}{|l|l|l|l|l|}
\hline$\widetilde{D}_{T}^{i} \times 10^{12}$ & Expt. data & $A^{a}$ & $B^{b}$ & $C^{c}$ \\
\hline & \multicolumn{5}{|c|}{$(0.200,0.200,0.600) \mathrm{wt} . \%$} \\
\hline THN & 0.90 & 1.08 & 1.35 & 0.41 \\
\hline IBB & 0.28 & 0.54 & -0.05 & -0.48 \\
\hline C12 & -1.18 & -1.62 & -1.30 & -0.07 \\
\hline & \multicolumn{5}{|c|}{$(0.303,0.307,0.390) \mathrm{wt} . \%$} \\
\hline THN & 1.09 & 1.32 & 1.79 & 0.62 \\
\hline IBB & 0.14 & 0.48 & -0.38 & -0.70 \\
\hline C12 & -1.23 & -1.80 & -1.42 & 0.08 \\
\hline & 1.1 & $(0.333,0.333,0.333) \mathrm{wt} . \%$ \\
\hline THN & 0.13 & 1.34 & 1.88 & 0.95 \\
\hline IBB & -1.23 & 0.41 & -0.51 & -0.50 \\
\hline C12 & \multicolumn{5}{|c|}{-1.75} & -1.37 & -0.45 \\
\hline & 1.14 & $(0.600,0.200,0.200) \mathrm{wt} . \%$ & \\
\hline THN & -0.23 & 1.40 & 2.00 & 0.67 \\
\hline IBB & -0.91 & -0.054 & -0.70 & -0.74 \\
\hline C12 & \multicolumn{5}{|c|}{-1.34} & -1.30 & 0.07 \\
\hline
\end{tabular}

${ }^{a}$ Firoozabadi-Ghorayeb-Shukla model (Firoozabadi et al, 2000).

${ }^{\mathrm{b}}$ Kempers Mass-Based model (Kempers, 2001).

${ }^{\mathrm{c}}$ Non-equilibrium thermodynamics, (Eslamian et al., 2010b).

Table 3 Thermodiffusion factors $\tilde{\alpha}$ for C12-THN-IBB-C12DMCH quaternary mixture at $(0.266,0.333,0.333,0.066) \mathrm{wt} . \%$ at $T=308 \mathrm{~K}$ and $P=5 \mathrm{MPa}$. Original experimental data were multiplied by $x_{i}\left(1-x_{i}\right)$ in order to make it compatible with the definition used here $\left(\sum_{i=1}^{4} \widetilde{\alpha}_{i}=0\right)$.

\begin{tabular}{|l|l|l|l|l|}
\hline$\widetilde{\alpha}$ & Expt. data & $A^{a}$ & $B^{b}$ & $C^{c}$ \\
\hline C12 & -0.36 & -1.53 & -0.62 & -0.13 \\
\hline THN & 0.23 & 1.27 & 0.71 & 0.16 \\
\hline IBB & -0.08 & 0.30 & -0.06 & 0.61 \\
\hline C12DMCH & 0.21 & -0.04 & -0.04 & -0.64 \\
\hline
\end{tabular}

${ }^{a}$ Firoozabadi-Ghorayeb-Shukla model (Firoozabadi et al, 2000).

${ }^{\mathrm{b}}$ Kempers Mass-Based model (Kempers, 2001).

${ }^{\mathrm{c}}$ Non-equilibrium thermodynamics, (Eslamian et al., 2010b).

\section{ASSOCIATING MIXTURES}

Aqueous mixtures of alcohols and mixtures of hydrocarbons and alcohols are good examples of associating mixtures due to the presence of the hydrogen bonds between the like and unlike molecules, whereas for instance mixtures comprised of linear hydrocarbons are good examples of non-associating mixtures. In non-associating mixtures, regardless of the mixture concentration, one component is often segregated on one side of the mixture (cold or hot side), whereas in associating mixtures, species concentrating on the hot side my start concentrating on the cold side if the mixture concentration changes. Spectroscopy studies on the molecular structures of associating mixtures reveal a very complex structure for such mixtures (Eslamian and Saghir, 2009c and references therein). Cluster size, composition and arrangement changes with mixture composition and temperature and their gradients. These structural changes have a very significant effect on the response of species to a temperature gradient and no wonder that the sign of thermodiffusion factors change in associating mixtures, when the temperature and concentration changes. 


\subsection{Experimental studies on associating mixtures}

While there are basically no experimental data on ternary or higher multicomponent associating mixtures, experimental data on binary associating mixtures are rather abundant. In near future, new experiments have been designed by the European and Canadian Space Agencies on alcohol and hydrocarbon ternary mixtures of interest, to be performed onboard the International Space Station. Owing to a sign change in thermodiffusion factor in associating mixtures with a change in mixture composition (concentration) and temperature, experiments on such mixtures are not as easy to perform as those of hydrocarbon mixtures, and no wonder that earlier data and still some of the present data are dubious.

Some of the major experimental works on the associating mixtures are as follows: Wiegand and coworkers (Ning and Wiegand, 2006; Wiegand et al., 2007; Polyakov and Wiegand, 2008) while performing experimental and theoretical studies on associating mixtures noticed that the thermodiffusion factor vanishes at a concentration close to the concentration at which the water hydrogen bonds break down. This was in fact an important finding also followed and reinvestigated by others (Eslamian and Saghir, 2009c).

Due to the complex nature of the associating problems and the lack of theoretical models, molecular dynamics (MD) simulation approach provides a suitable research tool for study of such mixtures. The MD simulation was originally conceived within theoretical physics, but is applied today mostly in materials science. In one study, MD simulation approach combined with a non-equilibrium algorithm that allows maintaining a constant heat flow through the system and another algorithm that accounts for the molecular interactions such as the hydrogen bonds was used (Rousseau et al., 2004), where a good agreement between the methanol-water experimental data and the MD simulations was observed including a sign change.

To investigate this problem in the molecular level, Rousseau et al., (2004) used the model of Prigogine and coworkers (1950), which is based on the Eyring's rate processes theory and the activation energy of viscous flow concept. By varying the particle-particle interaction parameters in that model, they realized that the slope of the thermodiffusion factor may change with concentration from positive to negative and a sign change may occur. Nieto-Draghi et al., (2005) employed a boundary-driven MD method in which there is no need to estimate the phenomenological coefficients in order to find thermodiffusion coefficients. Their simulations are successful in predicting a sign change in the studied mixtures.

Here are some examples of the recent experimental data: Platten et al., (2007) have repeated some of the experimental studies on the aqueous mixture of some alcohols in an attempt to eliminate the dubious data. Königer et al., (2009) measured the ordinary and thermodiffusion coefficients of ethanol-water mixture for a broad range of temperature (from 10 to $60{ }^{\circ} \mathrm{C}$ ) and concentration. They expanded the database of Kolodner et al., (1988). Their database is particularly suitable for model development and verification. One of their findings is that the cross over occurs at a concentration, which is insensitive to the mixture temperature. This indicates that the molecular structure of ethanol-water mixture and the cluster size is more dependent on the concentration of the mixture rather than temperature, while in general, temperature is expected to have an effect on the molecular structure, hydrogen bonds and intermolecular forces, as well.

\subsection{Theoretical studies in associating mixtures}

The response of the species to a temperature gradient in an associating mixture is quite difficult to model. This is because molecules of different species form large clusters that act as a cage for the individual molecules. The question is how to combine these varying and temperature and concentration dependent complexities and develop a thermodiffusion model? Also, in principle, is it possible to devise a comprehensive model that can work for any and every associating mixture? What is known so far is that currently such a comprehensive model does not exist. We are not even close to having such a model. Nevertheless, attempts have been made to develop thermodiffusion models for associating mixtures as well.

The existing theory of nonequilibrium thermodynamics, Eq. (2), is applicable to any binary mixture, provided that the net heat of transport can be adequately modeled. Therefore, the challenge is to model the net heat of transport appearing in Eq. (2). From a practical point of view, it has been shown that in associating mixtures, the concentration at which the sign of the slope of mixture viscosity changes usually coincides with a sign change in the thermodiffusion coefficient (Eslamian and Saghir, 2009c). Eq. (4), which works fairly well on hydrocarbon mixtures, does not predict a sign change; however, a sign change may be predicted if activation energy of viscous flow of each component can be considered variable. Owing to the difficulties in modeling of the activation energy of viscous flow of a component in the mixture, the activation energy of viscous flow used in Eq. (4) is associated with the species in pure state and it only changes slightly with temperature. To take into account the variations of the activation energy of viscous flow of each species in the mixture, the following expression was proposed for a binary complex mixture (Eslamian and Saghir, 2009c):

$\alpha=T \frac{D_{T}}{D}=\frac{E_{1}^{v i s}-E_{2}^{v i s}}{x_{1}\left(\partial \mu_{1} / \partial x_{1}\right)} \frac{\partial \ln \eta_{m i x} / \eta_{0}}{\partial x_{1}}$

where $\eta_{0}$ is a reference viscosity to render the second term in Eq. (10) dimensionless. In non-associating mixtures, the derivative of the natural logarithm of mixture viscosity with respect to mole fraction is a constant number of order 1 , whereas in complex mixtures its magnitude and sign may change when the mixture composition changes in which case according to Eq. (10), the sign of the thermodiffusion factor changes when the sign of the derivative of natural logarithm of the mixture viscosity versus concentration changes. In associating mixtures of water-alcohols (Eslamian and Saghir, 2009c and references therein), hydrocarbon-alcohols and also benzene-cyclohexane (Eslamian and Saghir, 2012a and references therein) a similar trend is observed. The performance of Eq. (10) depends on the complexity of the mixture. While its performance for benzene-cyclohexane is remarkable (Figure 3 ), it fails to adequately predict the data of methanol-hydrocarbons for instance (Eslamian and Saghir, 2012a). In associating mixtures, the interfacial forces, intermolecular/cluster bonds, hydrogen bonds and other chemical effects are a strong function of mixture composition and temperature.

In addition to the model described above, which is rather extensively tested on various associating mixtures, the Morozov model (2009) was also tested on benzene-cyclohexane mixtures. Also, in another attempt, Pan et al., (2007) used the nonequilibrium thermodynamics approach of Shukla-Firoozabadi, but using matching parameters extracted from the experimental data at the infinite dilution limits. The model is successful in predicting the trend of the data for concentrations other than infinite dilution, but as mentioned it is not stand-alone as it needs infinite dilution experimental data as input.

Eslamian and Saghir (2009c) proposed a mechanism for species separation and segregation in thermodiffusion in associating mixtures. In non-associating mixtures, especially those with simple and rather spherical molecules, species migration is affected by molecular weight and size, moment and inertia of molecules, and the interaction between alike and unlike molecules is probably less significant. In contrast, in associating mixtures, molecular interactions and a continuous change in the molecular structure play an important role in separation of molecules (Figure 4). In Figure 4, separation of methanol-water due to a temperature gradient is depicted. A dimer structure for water clusters has been assumed. A portion of water and methanol molecules remain isolated and intact in the mixture, whereas the rest of the molecules form hybrid methanol-water clusters. In the presence of a temperature gradient, it is easier for the isolated molecules or clusters to move in the mixture towards either the cold side or the hot side. The response of the 
hybrid clusters, however, is more complicated. Application of opposing thermal and concentration forces on the clusters may cause the breakdown of the cluster, or the cluster may move towards one end or even remains in its place and intact. Also, note that the local temperature may have an effect on hydrogen bonds, as at higher temperatures hydrogen bonds break easier.

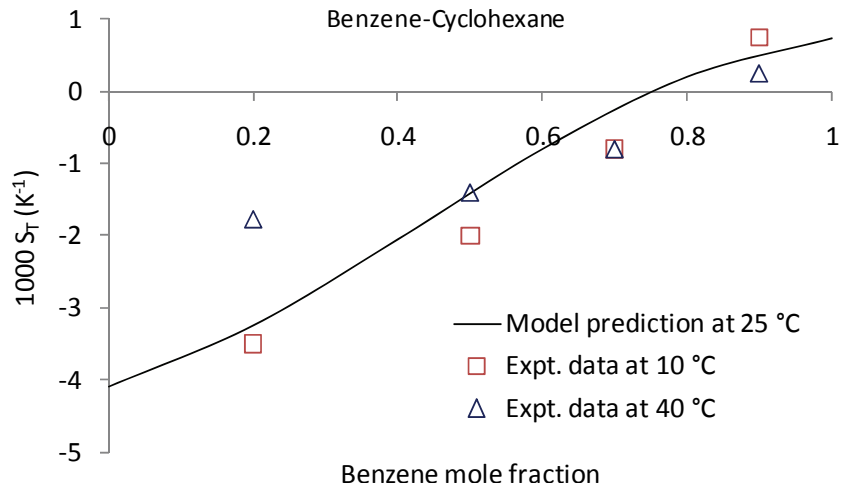

Fig. 3 Variation of Soret coefficient with concentration for benzenecyclohexane mixture. Model prediction (Eslamian-Saghir, 2012a) versus the experimental data (Debuschewitz and Köhler, 2001).

In ternary associating mixtures, there is very limited experimental and theoretical works. In a recent work, and in preparation for the near future space experiments on ternary mixtures of alcohols and hydrocarbon mixtures, Eslamian and Saghir, 2012c, proposed a semiempirical expression based on the linear nonequilibrium thermodynamics approach (Eq. 9) and the semi-empirical approach used for binary associating mixtures (Eq. 10). No comment can be made about the prediction power of the proposed expression due to the lack of experimental data. Other multicomponent models such as Firoozabadi and Kempers are not suitable for associating mixtures.

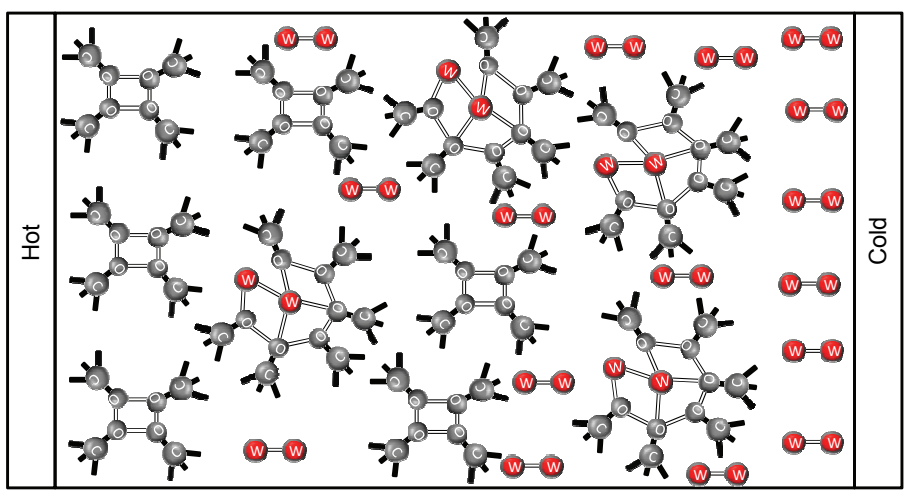

Fig. 4 Thermodiffusion in methanol-water mixture. Water clusters have been assumed as dimers and methanol clusters tetramers.

\section{MOLTEN METAL ALLOYS}

Investigation of thermodiffusion in molten metal mixtures, also referred to as thermotransport and thermomigration, was initiated by its potential application in the isotopic enrichment of materials (Otto, 1969), design of nuclear reactors that experience high temperatures (Sugisaki et al., 1981), and other nuclear-related activities (Bhat and Swalin, 1972; Bhat et al., 1973). Earlier thermodiffusion experiments on molten metal mixtures were performed on ground surface and in the presence of gravity. However, due to the detrimental effect of gravity on the concentration profile in some liquid metal mixtures, which may obscure the effect of thermodiffusion due to mixing, experiments were designed and performed in the microgravity environment during several space missions (Malmejac and Praizey, 1984; Praizey, 1986, 1989; Praizey et al., 1995; Van Vaerenbergh et al., 1998).

Thermodiffusion in molten metal mixtures has similarities and differences compared to simple hydrocarbon mixtures, as a reference. The differences arise from a different and unique molecular structure in molten metal mixtures, which are comprised of metal ions and free electrons. Compared to hydrocarbon mixtures, the molten metal mixtures have a more complex structure; however, compared to associating mixtures such as water-alcohols, have a simpler molecular structure. Thermodiffusion in such mixtures is due to ion-ion interactions, known as the extrinsic or ionic contribution and electronion interactions known as the intrinsic or electronic contribution. The ion-ion interaction between the ions of different constituent species is somewhat similar to the interaction between unlike molecules in a hydrocarbon mixture. Thus, any expression developed for nonassociating mixtures, such as Eq. (4), may be used to approximately predict the ionic contribution to the thermodiffusion factor. This is because Eq. (4) derived from the principles of the linear nonequilibrium thermodynamics, only accounts for the bulk interactions between molecules in a liquid mixture in which only temperature and concentration gradients are present. The presence of the free electrons in a molten metal mixture creates an electric field and electric force, not present in non-ionic mixtures.

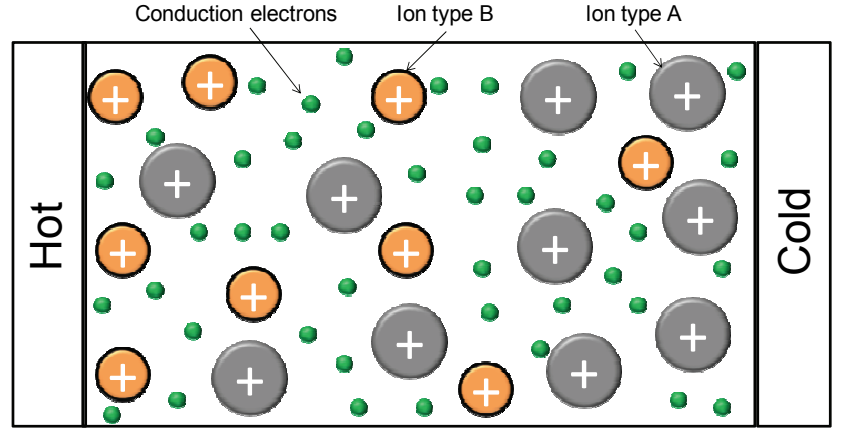

Thermal gradient force $\sim \nabla T$

Fig. 5 Schematic arrangement of ions and conduction electrons in a binary liquid metal mixture in steady state (zero mass flux). Large and small ions hove segregated on the cold and hot side, respectively.

Figure 5 shows the mechanism of separation in a binary molten metal mixture. The general description of the separation mechanism in hydrocarbon mixtures, described in the introduction section applies here as well as some additional effects due to the presence of the conduction electrons. The thermal forces push the free electrons towards the cold side as they conduct heat. This results in a concentration gradient in the number density of electrons in the mixture. The non-uniform distribution of electrons within the mixture, and also, to some extent, the non-uniform distribution of the metal ions, create an internal electric field that exerts forces on the particles through the thermoelectric effect. Assuming that the mixture forms an open circuit, such that in steady state there will be no flux of electrons, the electronic forces are mainly due to an induced internal electric field owing to the non-uniform distribution of electrons and ions within the mixture. Electronic forces acting on ions may assist or oppose the thermal and concentration forces.

The forgoing separation mechanism is for a given mixture composition. With a change in the mixture composition, the mixture properties, such as viscosity and thermoelectric power, may change as well. This may cause a complete rearrangement of all particles due to a possible change in the magnitude and even direction of concentration and electronic forces. In several mixtures, such as Na-K this change 
may be so intense that the species enriched on the cold side, in a given composition, now migrate to the hot side for the new composition.

A review of the existing research work on the Soret effect in molten metal alloys reveals a great deal of inconsistency and controversy. Despite the similarity of thermodiffusion in molten metal mixtures with that in other mixtures, thermodiffusion analysis in metal mixtures has been treated rather differently. For instance, the net heat of transport is defined rather differently and not in accordance with the nonequilibrium thermodynamics, i.e., Eq. (3). This has caused a great deal of ambiguity in thermodiffusion literature of molten metal alloys. Some of the sources of confusion were identified and discussed and an unambiguous theoretical model was proposed based on the linear nonequilibrium thermodynamics by Eslamian et al., (2010a). The proposed model considers the thermodiffusion factor in terms of the net heats of transport of two separate effects: the electronic and ion-ion thermal interactions. Owing to the similarity of the thermally induced mass diffusion with viscous flow, in that they are both activated processes, the ion-ion net heat of transport of each component was replaced by the activation energy of viscous flow of the same component in pure state. The electronic net heat of transport was estimated using an expression for the forces that act on an ion by the conduction electrons. The combination of the aforementioned modeling approach resulted in an expression that may be readily used for the estimation of thermodiffusion factors in binary liquid metal mixtures. On account of the electric field imposed by the free electrons in a molten metal mixture, it is rational to define a new net heat of transport, $q_{k}^{*}$. This argument results in the following modified expression for the thermodiffusion factor of component 1 in a liquid metal alloy:

$\alpha=\frac{Q_{1}^{*}-Q_{2}^{*}}{x_{1}\left(\partial \mu_{1} / \partial x_{1}\right)}+\frac{q_{1}^{*}-q_{2}^{*}}{x_{1}\left(\partial \mu_{1} / \partial x_{1}\right)}$

The net heat of transport due to ionic effects may be replaced by the activation energy of viscous flow in a fashion similar to that in hydrocarbon mixtures discussed before, i.e., Eq. (4). The electronic net heat of transport per mole of species $i$, may be related to the force acting on a solute ion by conduction electrons. Several workers, such as Galina and Omini, (1972) and Gerl, (1967) have proposed expressions for the electronic force. However, several parameters have to be known before those expressions can be used. Barker and Jones, (1984) derived more practicable expressions for the contribution of electronic or intrinsic forces on the overall thermodiffusion in liquid metal alloys. Using Barker and Jones results combined with Eq. (11), Eslamian et al. (2010a) proposed the following expression for the thermodiffusion factor in some liquid metal alloys:

$\alpha=\frac{E_{1}^{v i s}-E_{2}^{v i s}}{x_{1}\left(\partial \mu_{1} / \partial x_{1}\right)}+\frac{-\mid e\left(\left(z S_{1}-z_{1} S\right) T N\right.}{x_{1}\left(\partial \mu_{1} / \partial x_{1}\right)}$

where $\mathrm{N}$ is the Avogadro's number, $|e|$ is the absolute value of an electron charge, $z_{i}$ is the valence of ion $i, z$ is the mean valence of the ions in the mixture, and $S_{i}$ is the thermoelectric power of ions of type $i$, and $\mathrm{S}$ is the mean thermoelectric power of the mixture. Depending on the metal alloy characteristics, such as the mixture structure, concentration and temperature, the thermal and electronic forces acting on the ion of type $i$ may be in the same or opposite directions, i.e. the two terms on the right hand side of Eq. (12) may have the same or opposite signs. Performance of the proposed expression has been satisfactory when applied to those mixtures that are composed of components with comparable melting points, such as $\mathrm{Sn}-\mathrm{Zn}, \mathrm{Sn}-\mathrm{Pb}, \mathrm{Sn}-$ $\mathrm{Cd}$, etc. Although somewhat speculative, it was shown that the model fundamentally and qualitatively is able to predict a cross-over in the direction of the composition-dependent migration of $\mathrm{Na}$ and $\mathrm{K}$ components, consistent with the experimental observations. At low concentrations of $\mathrm{Na}, \mathrm{Na}$ migrates to the hot side, whereas at high concentrations, it moves to the cold side (Barker and Jones, 1984). The sign change in the thermodiffusion factor in Na-K mixture was attributed to the anomalous behavior of $\mathrm{Na}-\mathrm{K}$ mixture thermoelectric power and its effect on the relative magnitude and the sign of the electronic contribution with respect to the ion-ion contribution. Additional research is required to identify and address other aspects of this complex phenomenon and investigate the validity of the proposed speculative hypothesis for the sign change in Na-K mixture.

Note that while some of the experimental data and practical applications concern the Soret effect in metal isotopes, Eq. (12) only applies to thermodiffusion in a mixture of two different metals, as for isotopes of a metal, it is not easy to define two different activation energies of viscous flow. In a study somewhat pertinent to molten metal alloys, Dominguez et al. (2011) studied the Soret effect in silicate melts that causes the separation of metals and isotopes from the melt. They proposed a model and expression for the Soret coefficient in silicate mixtures based on the concept of activation energy that showed a good prediction power. We leave the evaluation of their model to a future publication. In a recent work, Srinivasan and Saghir (2012) have used the concept of the neural networks, to predict the thermodiffusion coefficients in molten metal mixtures. In this rather statistical and engineering approach, the existing experimental data are used to train a network, which can be later used to predict the thermodiffusion coefficients of other similar mixtures. The method was also used to study the relative weight and importance of each effect such as the physical properties of the species on the thermodiffusion coefficient.

\section{MOLTEN SEMICONDUCTOR+METAL MIXTURES}

Another relevant and very interesting problem that has potential technological applications in microelectronics and microelectromechanical devices and systems is thermodiffusion in a binary molten metal and semiconductor mixture. Thermodiffusion in molten metal-semiconductor mixtures has similarities with that in molten alloys.

Thermodiffusion is present in the temperature gradient zone melting (TGZM) method used for the fabrication of semiconductor devices and solar cells. In the TGZM method (Figure 6), a liquid zone (usually a molten substance, single or multicomponent), initially formed on a solid thin film such as silicon diffuses through the film under the influence of a temperature gradient applied perpendicular to the direction of the mass diffusion. The moving molten zone is in fact similar to a stationary layer, in a velocity frame of reference attached to the molten zone. The top surface of the wafer is heated, for instance, using heat radiation from the top; heat is removed at the bottom surface in order to help a temperature gradient to be established across the wafer. The film temperature should be high enough to produce a moving aluminumsilicon molten zone on the cold surface. Once the metal (aluminum) is melted, it dissolves some silicon forming a molten aluminum-silicon zone on the cold surface. Silicon in solid state in contact with the molten aluminum is dissolved in aluminum to form a molten siliconaluminum zone. Silicon atoms dissolved in aluminum move downward towards the lower end of the melting zone, which is in contact with a solid phase. At this interface, a dilute mixture of aluminum in silicon crystallizes and leaves a solid aluminum-doped silicon zone behind, while the melting zone continues to migrate upward.

Since the invention of the TGZM method by Pfann (1969), several workers have experimentally and mathematically analyzed the melting zone (usually using a moving boundary approach), e.g., (Wernick, 1956; Tiller, 1963; Cline and Anthony, 1976a, 1976b, 1977; Lozovskii and Popov, 1983). In his experiments, Wernick measured the penetration rate of molten aluminum or gold into bulk silicon or germanium. Anthony and Cline systematically studied thermodiffusion in the TGZM process in various systems and geometries such as droplet migration through films (e.g., aluminum droplets through silicon). They also used this technique to fabricate $\mathrm{p}-\mathrm{n}$ junctions in aluminum-doped silicon films. 
Rudolph et al., (1996) analyzed experimentally and theoretically the crystal growth of several semiconductors, such as GaAs, InP, PbTe and $\mathrm{CdTe}$, and also $\mathrm{Bi}-\mathrm{Pb}$ alloy in the melt zone and substantiated the important effect of thermodiffusion in the convection-less microgravity experiments. The thermodiffusion coefficient in Boeck and Rudolph (1986) is considered as an input parameter to the equation obtained for the growth or migration velocity. The authors have used the Winter and Drickamer (1955) model, proposed for metal systems to estimate the thermodiffusion factor. Chien and Mattes (1983) used the TGZM method to produce thin semiconductor layers of GaAs and InP through liquid-phase epitaxial growth (LPE). In this method, a moving substrate is in contact with the melting zone where a thin layer of a semiconductor is continuously deposited on the substrate.

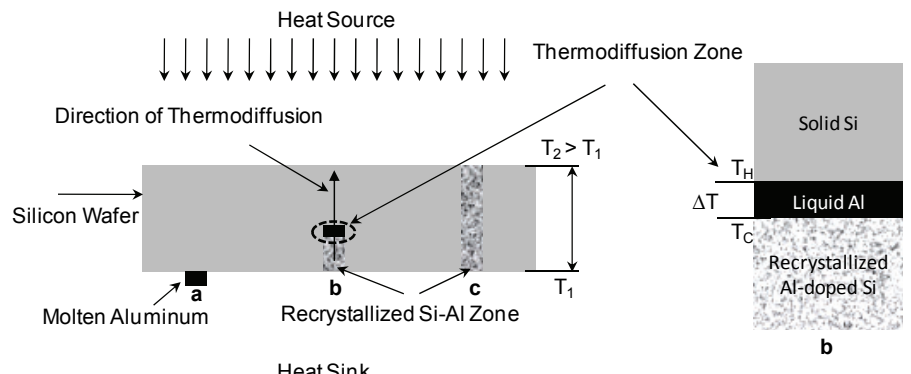

Fig. 6 Thermodiffusion in the Temperature Gradient Zone Melting (TGZM) method; (a) beginning of the process where an aluminum layer is melted due to the high surface temperature of the cold surface $\mathrm{T}_{\mathrm{C}}$; (b) as the molten aluminum dissolves silicon, it migrates towards the hot surface $\mathrm{T}_{\mathrm{H}}$, leaving behind a recrystallized column; (c) an aluminum-doped silicon column is formed extending from the cold side to the hot side of the silicon wafer; on the right side of the figure, the magnified thermodiffusion zone (b) is shown.

\subsection{Some applications of thermodiffusion in semiconductor industry}

At least two reviews of the applications of thermodiffusion in microelectronic industry, MEMS and solar cells are available (Eslamian and Saghir, 2012d; Buchin and Denisenko, 2006). Below, some of the proposed applications are reviewed. We note that the success of a patent or proposed application depends on how cost-competitive the method is compared to other possible methods. For some methods it may take years to become attractive and cost-effective.

\section{$\mathrm{P}-\mathrm{N}$ junction isolation}

P-n junctions are made in semiconductors to increase the breakdown voltage and reduce the leakage current in high power devices. It may also be interpreted as a method to isolate electronic components in an integrated circuit or a MEMS device by surrounding the components by $\mathrm{p}-\mathrm{n}$ or $\mathrm{n}-\mathrm{p}-\mathrm{n}$ junctions. Thermomigration has been suggested for the formation of $\mathrm{p}-\mathrm{n}$ junctions in the entire body of a silicon surface. It is a rapid and low thermal budget process.

\section{Fabrication of channels, trenches and conductive passages in semiconductors}

The development of high aspect ratio micromachining processes provides opportunities for the extension of three dimensional technologies that may be used for the integration of passive and active electronic devices and sensors. Among several existing methods, deep reactive ion etching (DRIE) with halide containing gases, and etching in alkali solutions are currently the two dominating silicon anisotropic etching techniques. However due to the cost and several other restrictions of these methods, thermomigration-based processes for the formation of trenches is also considered as an alternative (Gautier et al.,
2006). Fabrication of through-channels in semiconductors using the thermomigration method has basically two main steps: first to form a pdoped region within an n-type semiconductor with any desired shape, and then to use an etchant so as to remove the formed region without affecting the main body of the semiconductor. The formation of conductive channels that provide electrical connection between two components is another practical application of thermomigration in semiconductor devices.

\section{Fabrication of three-dimensional arrays in semiconductors}

Thermomigration has been also used to produce an array of silicon sharp needles or spikes that may be used for cortical penetration (Figure 7). To form an array, pads of aluminum on a silicon substrate are thermomigrated through an n-type silicon block. This leaves behind trails of p-doped channels isolated from each other by opposing $p-n$ junctions. A combination of mechanical and chemical techniques may be used to remove the regions between the thermomigrated columns and expose the needles. The needles need to be post-treated to create active electrode areas and contact pads (Normann et al., 1994).

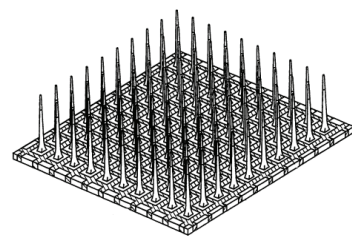

Fig. 7 3D array of p-type needles made by thermomigration of $\mathrm{Al}$ pads in the Si block (Normann et al., 1994).

\section{Fabrication of solar cells}

Some materials such as semiconductors exhibit the photovoltaic effect that causes them to absorb photons of light and release electron-hole pairs. When light energy strikes the solar cell surface, electrons are knocked loose from the atoms in the semiconductor material. If the material is treated or doped properly to prevent electron-hole recombination, and electrical conductors are attached to the positive and negative sides, an electrical circuit is formed and the electrons can be captured in the form of an electric current. This is the operating principle of silicon solar cells compared to thin film solar cells. For silicon solar cells, the bulk silicon substrate is generally doped p-type and the emitter layer is generally a thin, heavily-doped, $\mathrm{n}^{+}$layer that is formed in an emitter diffusion step by solid-state diffusion of phosphorus at elevated temperatures. Thermodiffusion may be used for the doping process, similar to the formation of a $p-n$ junction described before (Anthony et al., 1976).

\section{Material property manipulation in MEMS and NEMS devices}

A new emerging application of thermodiffusion in micro- and nanoelectronic devices is by changing the materials properties for a particular application, such as in the fabrication of silicon micromechanical resonators. Silicon micromechanical resonators can enable a cost-effective integrated platform for timing, wireless connectivity and multi-band spectral processing. To achieve stable low-phase-noise frequency references in silicon micromechanical resonators, the temperature coefficient of frequency (TCF) of silicon may be compensated without compromising on the quality factor of the resonator, using doping through changing the number of free charge carries in a silicon wafer, using thermodiffusion of aluminum into silicon (Samaro et al, 2009, 2010).

\subsection{Modeling of thermodiffusion in molten semiconductor- metal mixtures}


Earlier works have somewhat studied thermodiffusion in the context of the temperature gradient zone melting method, e.g., (Lozovskii and Popov, 1983; Boeck and Rudolph, 1986). There is no tailored model or expression for the thermodiffusion factor in molten semiconductormetal mixtures. Recently, thermodiffusion and convection was studies by Eslamian and Saghir (2011) in a thin molten layer. They used Eq. (4) to estimate thermodiffusion factor required as in input to solve the transport equations. They studied the presence and importance of remixing effects due to convection in a thin layer. The results have shown that in a micro-layer, convection is weak and perhaps has no adverse effect. Rayleigh-Benard instability in layers heated from below and above is an important research topic when thermodiffusion for instance in thin films and thermogravitational columns is concerned. Review of papers concerning the instability is not the focus of the present work. Some classic and recent works on instability include but are not limited to the following: (Legros et al., 1975; Henry and Roux, 1986; Zimmermann et al., 1992; Larre et al., 1997; Platten et al., 2007; Zebib, 2007, Ryzhkov and Shevtsova, 2009). The objective of the instability analyses is to find a critical Rayleigh number for the onset of convection in various geometries. Recently, Gusev et al. (2011) studied the dynamics of thermodiffusion in a transient system by solving the transport equations in a general binary system assuming a constant value for thermodiffusion factor. The objective of that work was to study the distribution of species by solving the transient species equation rather than estimating the thermodiffusion factor.

In the analysis performed by Eslamian and Saghir (2011) the molten zone is assumed to be in stationary state to avoid a moving boundary problem and the thermodiffusion factor is obtained using Eq. 4. One challenge to estimate the thermodiffusion coefficient is to calculate somehow the ordinary diffusion coefficient. There is very limited experimental or analytical work on ordinary diffusion coefficients in molten semiconductor-metal mixtures. Also, there is no equation of state to be used to estimate the derivative of chemical potentials in the case of non-ideal mixtures.

\section{POLYMER AND DNA MIXTURES}

\subsection{Thermophoresis in polymer mixtures}

While in some mixtures such as hydrocarbons, alcohol-water and molten metal alloys, all constituent species have comparable molecular sizes, are miscible, and are in the liquid phase, thermodiffusion is also realized in mixtures comprised of a main liquid known as the solvent and a dispersed phase in the form of nanoparticles, colloidal particles, and macromolecules of polymers or DNA and so on. Thermodiffusion of a dispersed phase in a fluid is also called thermophoresis. While thermophoresis covers a broad area including emerging research on nanoparticle and nanofluids (Martin and Bou-Ali, 2011) and is applicable to several types of mixtures, here we limit our discussion to the polymer and DNA mixtures, leaving other topics such as thermophoresis of charged colloids to the experts in those fields.

Thermodiffusion experimental data in polymer solutions have been available since 1950s, e.g., (Emery and Drickamer, 1955; Whitmore, 1960; Schimpf and Giddings, 1989). Schimpf and Giddings (1989) also provide a good review of previous experimental and theoretical works. The main conclusion of those early studies is that the thermodiffusion coefficient in long-chain polymer solutions is almost independent or less-dependent on the polymer molecular weight. More recent works e.g., (Stadelmaier and Köhler, 2008, 2009; Chan et al., 2003) have expanded the previous works on long-chain polymer solutions to shortchain polymer solutions, as well. Stadelmaier and Köhler experimental data show that while in a short-chain polymer solution, polymer molecules may migrate to the hot side, with the increase of the polymer molecular weight a cross-over may occur causing the polymer molecules to enrich on the cold side. A negative Soret coefficient is predicted by reverse nonequilibrium molecular dynamics (Zhang and Müller-Plathe, 2006), and the lattice model of Luettmer-Strathmann
(2005). Negative Soret coefficient has been observed in other polymer solutions, as well, e.g., poly(ethylene oxide) in ethanol-water solution (de Gans et al., 2003).

To understand the mechanism of the separation of polymer and DNA molecules from the solvent, we start with a brief description of the dynamics of such mixtures. It is noted that DNA molecules repeat their building block or base and therefore may be safely treated by the same way the polymer molecules are treated. The study of the dynamics of polymers in a liquid is not restricted to thermophoresis and has been advanced significantly in the area of hydrodynamics of polymer and DNA solutions (Larson et al., 1997). The most commonly used physical and mathematical models of polymer flow in the liquid phase are the freely jointed bead-rod and bead-spring models. The beads represent frictional drag centers connected by rods or springs. Three major forces that act on a flowing polymer bead in a dilute solution include the drag, spring, and Brownian forces, e.g., (Bird et al., 1987).

Once a temperature gradient switches on, while initially both species are forced to move in the direction of the heat flow, with the establishment of a developing concentration gradient in the mixture and the interaction of thermal, concentration and drag forces, the more thermophobic component is pushed toward the cold side, whereas the other molecule has to move to the hot side. In steady state condition, thermal and concentration forces are acting on the molecules, whereas before the steady state is established, the diffusion drift velocities are non-zero and drag forces also work on the molecules. Once the segregation process is triggered and molecules attain a maximum drift velocity, the thermal forces are counteracted by the drag and other forces, such as the Brownian forces. A concentration is established gradually with time, leading to a concentration-induced diffusion flow opposite to the thermodiffusion-induced flow, and this causes a decrease in the overall drift velocity and drag forces (Eslamian and Saghir, 2010d). Villain-Guillot and Würger (2011) argued that the Soret motion in binary liquids arises to a large extent from rectified velocity fluctuations around molecules. Using a hard-bead model, they derived a net force on each molecule, which is proportional to the temperature gradient and depends on the ratio of the molecular masses and moments of inertia.

The thermodiffusion models applicable to polymer solutions are discussed by Hartung et al. (2006). Würger (2009) derived a formula for the thermodiffusion coefficient, which is comprised of two terms with opposite signs. In the limit of the high polymer molecular weight, the first term vanishes and the Stokes hydrodynamic term prevails, whereas in the short-chain polymer solution, the magnitude of the two terms may become comparable and a sign change in the thermodiffusion coefficient may occur. This formula, however, could only qualitatively predict a sign change and no comparison with the experimental data was provided. What is understood from the hydrodynamics of polymers is that if the polymer concentration is very low, the beads or segments of a long-chain polymer molecule move within the solvent almost independent of one another. If this is the case, the polymer is said to be in infinite dilution. This is also the basis of the Würger (2009) model, which assumes that the motion of a complex polymer molecule may be simulated by the motion of individual beads of the polymer molecule with an effective hydrodynamic radius. Recently, Bringuier (2011), using a Brownian and hydrodynamic approach, qualitatively studied the thermophoresis of linear polymer chains and argued that the effect is due to two forces, one stemming from the departure of the molecular-velocity distribution from Maxwell's distribution and the other owing to the dependence of viscous friction on the temperature and position of the molecules in the mixture. His model can potentially predict a sign change as the two effects have opposing signs. Runyon and Williams (2011) clarified and further developed the thermal flow-field fractionation method (ThFFF) applied to polyacrylates and compared their experimental results with the theories of Semenov and Schimpf (2004) and Mes (2003). They had to estimate the undetermined input parameters in those theories. The agreement with the experimental data was fair. 
The rational approach to model thermodiffusion in polymer mixtures is the hydrodynamics approach. However this approach does not provide a practicable expression yet. One successful approach is the linear nonequilibrium thermodynamics, Eq. (2), if the net heat of transport in polymer mixtures can be modeled adequately (Eslamian and Saghir, 2010d). In their approach, it was assumed that the net heat of transport of the solvent molecules equals the activation energy of viscous flow of the solvent in the pure state, i.e. $Q_{S}^{*}=E_{S}^{v i s}$. Simulation of the net heat of transport of the polymer molecules, on the other hand, is more challenging, as polymers are available in various chain lengths and therefore different molecular weights and viscosities. Polymers although soluble in solvents and forming a liquid mixture, are in the solid state at room temperature. Therefore, it is not possible to estimate the activation energy of viscous flow of a polymer at a mixture temperature, which is usually the room temperature. Emery and Drickamer (1955) argued that the viscosity data of a given polymer solution at low concentrations may be used to calculate the activation energies of viscous flow of the solution at various concentrations. Then the activation energy of viscous flow of the polymer (at the limit where no solvent is present in the solution) may be estimated by linear extrapolation of the solution activation energies with respect to the polymer concentration. This may give a reasonable estimate of the polymer activation energy of viscous flow and the net heat of transport; however, viscosity data are required for various polymer-solvent combinations at various polymer molecular weights. Emery and Drickamer (1955), therefore, proposed a universal number for the activation energy, which is not accurate enough bearing in mind that different combinations of polymer-solvent may have very different activation energies. Eslamian and Saghir assumed that the activation energy of viscous flow of a long-chain polymer molecule is proportional to the activation energy of viscous flow of the monomers (that represent the moving segments) of that polymer, usually in the liquid phase. They proposed the following equation for the activation energy of viscous flow of a polymer molecule, $E_{P}^{v i s}$ :

$$
E_{P}^{v i s}=E_{M}^{v i s} \operatorname{Ln}\left(\frac{M_{P}}{M_{B}}\right)^{a}
$$

where $E_{M}^{v i s}$ is the activation energy of viscous flow of the polymer monomer, $M_{P}$ and $M_{B}$ are the molecular weights of the polymer and the polymer moving segment or bead, respectively. The values of $a$ is characteristics of the polymer-solvent system. For most flexible polymers, parameter $a$ in Eq. (13) varies between 0.5 and 0.8 .

The molecular weight of the moving segment is close to the molecular weight of the monomer. The following expression has been proposed for the Soret coefficient of an ideal polymer solution in the infinite dilution limit:

$$
S_{T}=\frac{E_{M}^{v i s} a L n\left(\frac{2 M_{P}}{M_{M}}\right)-E_{S}^{v i s}}{R T^{2}}
$$

where the bead molecular weight $M_{B}$ has been assumed to be half of the monomer molecular weight, $M_{M}$. Eq. (14) is semi-empirical, but has a fair prediction power compared to other models (in fact there is no other expression) while it involves readily obtainable parameters. A negative thermodiffusion coefficient was predicted by the model for the short-chain polymer solutions of polystyrene in cyclooctane and tetrahydrofuran, consistent with the experimental data. The model also correctly predicts that with the increase of the polymer molecular weight, the sign of the Soret coefficient may change and at high polymer molecular weights, the thermodiffusion coefficient and the
Soret coefficient approach a plateau, although they still logarithmically increase with the increase of the molecular weight.

The theory developed here is most applicable to infinite dilute solutions because of the limitation in the modeling of the net heat of transport. For more general cases, the nonequilibrium thermodynamics approach remains valid; however more sophisticated approaches and modifications are required on account of the complex structure of polymer solutions. Perhaps other chemical and physical characteristics of the polymer may have an effect on the thermodiffusion and Soret coefficients.

\subsection{DNA mixtures}

Employment of a temperature gradient for DNA and virus manipulation (Blanco et al., 2011) is one of the recent applications of thermodiffusion. Thermophoresis is found as a good alternative to the currently employed electrophoresis technique, owing to its tune ability by adjusting the temperature gradient (Reineck et al., 2010; Duhr et al., 2004). Thermophoresis of DNA combined with convective flows may lead to accumulation of DNA and an increase in the local concentration of DNA molecules (Braun and Libchaber, 2002). It has been hypothesized that life might have originated by replication of DNA molecules in the rocks and porous precipitates in the oceans across which a temperature gradient is present (Braun and Libchaber, 2004).

Few experimental data are available on DNA solutions (Reineck et al., 2010; Duhr et al., 2004; Braun and Libchaber, 2002; Duhr and Braun, 2006; Iacopini et al., 2006). A variety of DNA molecules in different aqueous solutions have been used for thermodiffusion experiments by several research groups, making the comparison and model validation difficult. This is because thermodiffusion factor depends on the interfacial and intermolecular forces, and the presence of additives can alter the thermodiffusion coefficients.

In the absence of any thermodiffusion model, Eslamian and Saghir, (2012e) extended their model developed for polymer solutions, Eq. (14) to the case of DNA mixtures. For the modeling of DNA solutions compared to general polymer solution, despite close similarity, some other complications are involved as the base pair of DNA is not in the liquid phase and as such no viscosity or activation energy of viscous flow can be defined. It was assumed that the viscosities of single- and double- stranded DNA molecules are close to the viscosities of alkane molecules with comparable molecular weights. This assumption has been made owing to the similarities between the structure of alkanes and DNA. Besides, the viscosity data of alkanes required to estimate the activation energy of viscous flow are available. For the purpose of estimating the viscosity of double-stranded DNA with a molecular weight of about $660 \mathrm{~g} \cdot$ mole $^{-1}$, pentacontane $\left(\mathrm{C}_{50} \mathrm{H}_{102}\right)$ was chosen and for a single- stranded DNA molecule, tetracosane $\left(\mathrm{C}_{24} \mathrm{H}_{50}\right)$. The viscosity data of the aforementioned alkane molecules are available at temperature higher than the room temperature, adding more inaccuracies to the modeling approach.

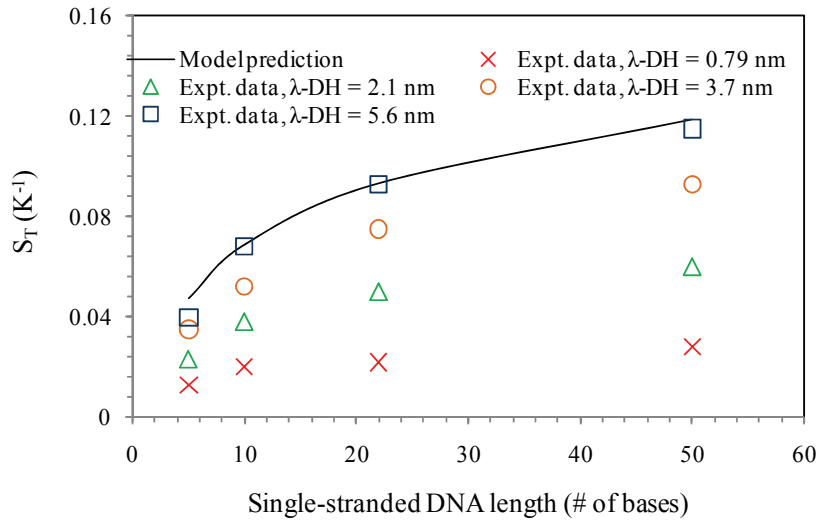

Fig. 8 Measured and estimated Soret coefficients of single-stranded DNA solutions at room temperature (expt. data from Reineck et al., 2010). 
The model is more successful on dilute single-stranded DNA solutions, due to the less-complex structure of such molecules compared to double-stranded DNA molecules. Figure 8 shows the measured and estimated Soret coefficients for single-stranded DNA solutions at room temperature. Experimental data in Figure (8) (Reineck et al., 2010) are associated with various Debye shielding lengths, being inversely proportional to the solution concentration. The data points associated with the largest Debye length, which are associated with the most dilute solution, shows an excellent agreement with the model predictions. Note that Eq. (14) in the present form has been derived for the infinite dilution limit.

In DNA solutions, a sign change in the Soret coefficient is also observed, when the mixture temperature changes (Blanco et al., 2011). This sign change is attributed to the interacting and interfacial forces between the solvent and the DNA molecules. Iacopini et al. (2006) proposed an empirical equation in the following form that fits the experimental data by aid of several fitting parameters and can predict a sign change when the mixture temperature changes:

$S_{T}=S_{T}^{\infty}\left[1-\exp \left(\frac{T^{*}-T}{T_{0}}\right)\right]$

where $S_{T}^{\infty}$ is the Soret coefficient in the high temperature limit, $T^{*}$ is the temperature at which the Soret coefficient is zero and $T_{0}$ is another fitting parameter. The sign change of the Soret coefficient with temperature has been attributed to the hydration entropy in water and a sign change in this entropy at a temperature of about $20^{\circ} \mathrm{C}$ (Duhr and Braun, 2006). At low temperatures, the hydration entropy can dominate thermodiffusion so that the molecules segregate on the hot side.

\section{CONCLUDING REMARKS}

The main challenge in the field is to try to congregate the various modeling approaches in an attempt to develop a comprehensive thermodiffusion model. The existing approaches, i.e., the nonequilibrium thermodynamics, the kinetic theory and stochastic approach and the hydrodynamic approach are the three main tracks, but cannot model the entire process alone due to the complex nature of thermodiffusion. Reliable experimental data are still scarce in some areas such as the ternary and higher multicomponent mixtures. Below, the challenges in each area are highlighted separately.

\subsection{Hydrocarbon mixtures}

In hydrocarbon mixtures, more experimental data are needed for ternary and higher multicomponent mixtures to expand the database. Of particular interest is to understand the effect of temperature and concentration on thermodiffusion coefficient of ternary mixtures. In binary mixtures, some other complex systems beside the benzenecyclohexane mixture is needed to be studied as well. Modeling of thermodiffusion in linear hydrocarbon mixtures, by far has been the most successful among binary liquid mixtures. However, still no model can adequately predict the thermodiffusion factors or coefficients. In ternary and higher multicomponent mixtures, there is no model that can predict the sign of the thermodiffusion coefficient of all three components in the mixture.

\subsection{Associating mixtures}

Experimental data on binary associating mixtures, such as aqueous solutions of alcohols and acetone are available covering a broad range of temperature and concentration. The challenge in this area is the modeling of the process and understanding of the mixture molecular structure. These mixtures have an exceedingly complex molecular structure that changes significantly when temperature and concentration changes. Development of a thermodiffusion model that can fairly predict the sign and magnitude of the thermodiffusion factors is demanding. A thorough understanding of the molecular structure, hydrogen bonds, interfacial forces, and the clustering mechanism in such mixtures is required before a model can be devised.

\subsection{Molten metal and semiconductor mixtures}

Thermodiffusion research on molten metal alloys has been more extensive and rigorous in the last couple of decades, perhaps due to its importance in isotopic separation of metals. From a fundamental point of view, the problem is not understood well yet due to the presence of the free electrons in the mixture and their contribution to the Soret effect. In some cases, e.g., Na-K mixture, a sign change also occurs when the concentration changes. Therefore, the challenge is to identify the relative contribution of ionic and electronic to the thermodiffusion factor and to study how these effects change with concentration.

In molten metal-semiconductor mixtures, the effect of free electrons on thermodiffusion factor is perhaps less profound compared to that in the metal-metal mixtures, but it is not absent. Reliable thermodiffusion data on metal-semiconductor mixtures is scarce due to the instability issues in such mixtures. Thermodiffusion, if controlled and predicted adequately, has shown to have potential applications in p-n junction formation and recently on the modification of the materials properties, among others. Future work is therefore focused on well-controlled and specialty applications. On the theoretical side, accurate experimental data for model development are lacking.

\subsection{Polymer and DNA mixtures}

Thermodiffusion in polymer and DNA mixtures falls more in the category of thermophoresis and therefore has been treated based on the hydrodynamics theory. In this review, however, we showed that the linear nonequilibrium thermodynamics approach is applicable to thermophoresis of DNA and polymer mixtures as well. Modeling of thermodiffusion in dilute polymer mixtures has been performed with various degrees of success, but it is still difficult to develop models for concentrated mixtures.

Manipulation of DNA molecules is of particular interest in the area of biological research. The challenge here is to quantify the DNA manipulation by a temperature gradient, something that needs a reliable model to predict the thermodiffusion data. Experimental data on DNA mixtures is insufficient and scattered. Using different types of DNA molecules and solvents makes the comparison between the results difficult. Systematic study of thermodiffusion in DNA mixtures is required.

\subsection{Molecular dynamics (MD) simulations}

Molecular dynamic simulations are like artificial or numerical experiments if the potential functions are defined and used properly. Performing reliable experiments in some cases is indeed difficult and expensive and therefore MD simulation is a good alternative. The interaction of ions and electrons in a mixture with like and unlike molecules is a complex phenomenon. Thermodiffusion process among other transport phenomena is considered to be the most sensitive to the interaction potential shape, at least in low density conditions. MD simulation has been employed to compute the Soret coefficients in some less-complex binary liquid mixtures such as aqueous mixtures, e.g., (Nieto-Draghi et al. 2005; Galliero and Montel, 2008; Perronace et al. 2002) and also associating mixtures (Rousseau et al. 2004). It is expected that in the future, MD simulations continue to serve as a good tool to investigate Soret effect in other complex systems, such as molten metal alloys, polymers, etc. This will help to better understand the thermodiffusion process in complex systems and help to develop or 
improve the existing physical and mathematical thermodiffusion models.

\subsection{Equation of state}

The availability of an Equation of State (EOS) or at least the availability of activity coefficient experimental data is crucial in evaluating many of the existing theoretical models used to estimate the thermodiffusion factor and coefficient. This is because in non-ideal mixtures, in particular, the variation of the chemical potential versus concentration is a strong function of mixture concentration and temperature. An EOS and even activity coefficient experimental data are lacking in some mixtures such as molten metal + semiconductor mixtures, DNA and polymer mixtures and some other mixtures. For hydrocarbon mixtures, however, as well as associating mixtures, equations of states are available. To our knowledge and personal experience, for the former, the Peng-Robinson and for the later the Perturbed-Chain Statistical Associating Fluid Theory (PC-SAFT) and Cubic Plus Association (CPA) equations of states have shown acceptable performance.

Also, in order to determine the thermodiffusion coefficients instead of the Soret coefficient and thermodiffusion factor, knowledge of the ordinary diffusion coefficient is essential. Ordinary diffusion coefficients or correlations and models that can estimate them may not be available for some mixtures such as the molten metal and semiconductor mixtures. Also, an EOS for the metal-semiconductor mixtures is still lacking.

\section{NOMENCLATURE}

$a \quad$ a coefficient of $\mathrm{O}(1)$ in Eq. (13) (-)

$A, B, C, D$ parameters in Eq. (9)

$c \quad$ mixture overall concentration $\left(\mathrm{mol} / \mathrm{m}^{3}\right)$

$D \quad$ ordinary diffusion coefficients $\left(\mathrm{m}^{2} / \mathrm{s}\right)$

$D_{T} \quad$ thermodiffusion coefficient in binary mixtures $\left(\mathrm{m}^{2} / \mathrm{s}\right)$

$\widetilde{D} \quad$ thermodiffusion coefficient in ternary mixtures $\left(\mathrm{m}^{2} / \mathrm{s}\right)$

$e \quad$ electron charge $(\mathrm{C})$

$E^{\text {vis }} \quad$ activation energy of viscous flow $(\mathrm{J} / \mathrm{mol})$

$\bar{H} \quad$ molar enthalpy $(\mathrm{J} / \mathrm{mol})$

j $\quad$ mass flux $\left(\mathrm{mol} / \mathrm{m}^{2} \mathrm{~s}\right)$

$\dot{j}_{q}^{\prime} \quad$ pure heat flux $\left(\mathrm{W} / \mathrm{m}^{2}\right)$

$L_{i j} \quad$ phenomenological coefficients

$M_{k} \quad$ molecular weight $(\mathrm{kg} / \mathrm{mol})$

$N \quad$ Avogadro's number (-)

$q^{*} \quad$ net heat of transport of electrons in Eq. (11) $(\mathrm{J} / \mathrm{mol})$

$Q^{*} \quad$ heat of transport $(\mathrm{J} / \mathrm{mol})$

$R \quad$ universal gas constant $(\mathrm{J} / \mathrm{molK})$

$\bar{S} \quad$ molar entropy $(\mathrm{J} / \mathrm{molK})$

$S \quad$ thermoelectric power

$S_{T} \quad$ Soret Coefficient (1/K)

$x \quad$ mole fraction (-)

$T \quad$ temperature (K)

$T^{*} \quad$ temperature at which the Soret coefficient is zero in Eq. (15) $(\mathrm{K})$

$T_{0} \quad$ fitting parameter in Eq. (15) (K)

$\bar{U} \quad$ partial molar energy $(\mathrm{J} / \mathrm{mol})$

$\bar{V} \quad$ partial molar volume $\left(\mathrm{m}^{3} / \mathrm{mol}\right)$

$z \quad$ valence of the electron (-)

Greek Symbols

$\alpha \quad$ thermodiffusion factor in binary mixtures (-)

$\tilde{\alpha} \quad$ thermodiffusion factor in ternary mixtures $(-)$ $\alpha_{0} \quad$ thermodiffusion factor on account of kinetic effects in Eq. (6) (-)

$\mu \quad$ chemical potential $(\mathrm{J} / \mathrm{mol})$

$\eta_{0} \quad$ a reference viscosity in Eq. (10) (Pas)

$\eta_{\text {mix }} \quad$ viscosity of the mixture (Pas)

$\tau \quad$ ratio of vaporization energy to activation energy in Eq. (7) $(-)$

Superscripts

$x \quad$ mole-based in Eq. (8)

vis viscous flow

$\infty \quad$ high temperature limit

Subscripts

$B$ bead

$k \quad$ component $k$

M monomer

$P \quad$ polymer

$S \quad$ solvent

\section{REFERENCES}

Abbasi, A., Saghir, M. Z., and Kawaji, M., 2011, "An improved thermodiffusion model for ternary mixtures using Fujita's free volume theory," Journal of Nonequilibrium Thermodynamics, 36, 259-272. http://dx.doi.org/10.1515/JNETDY.2011.016

Anthony, T. R. and Cline, H. E., and Winegar, D. M., 1976, "Solar cell", US3936319.

Barker, G. C. and Jones, W., 1984, "Thermal diffusion and thermoelectric powers in molten $\mathrm{Na}-\mathrm{K}, \mathrm{Na}-\mathrm{Rb}, \mathrm{K}-\mathrm{Rb}$ and $\mathrm{Ag}-\mathrm{Au}$,“ Journal of Physics, F: Metal Physics, 14, 437-447. http://dx.doi.org/10.1088/0305-4608/14/2/016

Bearman, R. J., and Kirkwood, J. G., 1958, "Statistical mechanics of transport processes. XI. Equations of transport in multicomponent systems," Journal of Chemical Physics, 28, 136. http://dx.doi.org/10.1063/1.1744056

Bhat, B. N., and Swalin, R. A., 1972a, "Thermal diffusion of tritium in $\mathrm{Nb}$ metal,“" Acta metallurgica, 20, 1387-1396.

http://dx.doi.org/10.1016/0001-6160(72)90074-0

Bhat, B. N., and Swalin, R. A., 1972b, "Thermotransport of cobalt in liquid silver," Scripta Metallurgica, 6, 523-527.

http://dx.doi.org/10.1016/0036-9748(72)90040-3

Bhat, B. N., Murarka, S. P., and Swalin, R. A., 1973, "Thermotransport of beryllium and mercury in liquid sodium," Scripta Metallurgica, 7, 523-527.

http://dx.doi.org/10.1016/0036-9748(73)90106-3

Bielenberg, J. R., and Brenner, H., 2005, "A hydrodynamic/Brownian motion model of thermal diffusion in liquids," Physica A, 356, 279-293. http://dx.doi.org/10.1016/j.physa.2005.03.033

Bird, R. B., Curtiss, C. F., and Armstrong, R. C. and Hassager, O., 1987,“ Dynamics of Polymeric Liquids, 2nd Edition, John Wiley \& Sons, New York.

Blanco, P., Bou-Ali, M. M., Platten, J. K., J.A. Madariaga, J.A., Urteaga, P., and Santamaría, C., 2007, "Thermodiffusion coefficient for binary liquid hydrocarbon mixtures," Journal of Non-Equilibrium Thermodynamics," 32, 309-317. http://dx.doi.org/10.1515/JNETDY.2007.023 
Blanco, P., Bou-Ali, M. M., Platten, J. K., de Mezquia, D. A., Madariaga, J. A., and Santamaría, C., 2010, "Thermal diffusion coefficients of binary and ternary hydrocarbon mixtures," Journal of Chemical Physics, 132, 114506.

http://dx.doi.org/10.1063/1.3354114

Blanco, P., Kriegs, H., Lettinga, M., P., Holmqvist, P. and Wiegand, S., 2011, "Thermal diffusion of a stiff rod-like mutant Y21M fd-virus," Biomacromolecules, 12, 1602-1609.

http://dx.doi.org/10.1021/bm2000023

Brady, J. B., 1975, "Reference frames and diffusion coefficients," American Journal of Science, 275, 954-983.

http://dx.doi.org/10.2475/ajs.275.8.954

Braun, D., and Libchaber, A., 2002, "Trapping of DNA by thermophoretic depletion and convection," Physical Review Letters, 89, 188103.

http://dx.doi.org/10.1103/PhysRevLett.89.188103

Braun, D., and Libchaber, A., 2004, "Thermal force approach to molecular evolution," Physical Biology, 1, P1-P8.

http://dx.doi.org/10.1088/1478-3967/1/1/P01

Brenner, H., 2006, "Elementary kinematical model of thermal diffusion in liquids and gases," Physical Review E, 74, 036306.

http://dx.doi.org/10.1103/PhysRevE.74.036306

Bringuier, E., 2011, "Gauge-invariant approach to thermodiffusion in a liquid binary mixture, Physcia A, 390, 1861-1875.

http://dx.doi.org/10.1016/j.physa.2011.01.027

Bringuier, E., 2011, "Thermophoresis of linear polymer chains," $C . R$. Mecanique, 339, 349-354.

http://dx.doi.org/10.1016/i.crme.2011.03.013

Boeck, T., and Rudolph, P., 1986, "The influence of thermal diffusion on the travelling heater method in a diffusion controlled growth system," Journal of Crystal Growth, 79,105-109

http://dx.doi.org/10.1016/0022-0248(86)90423-9

Buchin, E. Y., and Denisenko, Y. I., 2006, "Use of thermomigration in MEMS technology", Proceedings of SPIE; 6260: 62601L1-62601L10.

Chan, J., Popov, J. J., Kolisnek-Kehl, S., and Leaist, D. G., 2003, "Soret coefficients for aqueous polyethylene glycol solutions and some tests of the segmental model of polymer thermal diffusion," Journal of Solution Chemistry, 32, 197-214.

http://dx.doi.org/10.1023/A:1022925216642

Chien, C-P., and Mattes, B. L., 1983," Thermal Soret diffusion in the liquid phase epitaxial growth of binary III-V compounds, Journal of Vacuum Science \& Technology B, 1, 648-655.

http://dx.doi.org/10.1116/1.582571

Cline, H. E., and Anthony, T. R., 1976a, "High-speed droplet migration in silicon," Journal of Applied Physics, 47, 2325-2331. http://dx.doi.org/10.1063/1.323008

Cline, H. E., and Anthony, T. R., 1976b, "Thermomigration of aluminum-rich liquid wires through silicon, Journal of Applied Physics, 47, 2332-2336.

http://dx.doi.org/10.1063/1.323009
Cline, H. E., and Anthony, T. R., 1977, "Thermomigration of molten Ga in Si and GaAs, “Journal of Applied Physics, 48, 2196-2201. http://dx.doi.org/10.1063/1.324021

Colombani, J., Gallie'ro, G., Duguay, B., Caltagirone, J-P., Montel, F., and Bopp, P. A., 2002, "A molecular dynamics study of thermal diffusion in a porous medium," Physical Chemistry Chemical Physics, 4, 313-321.

http://dx.doi.org/10.1039/b106800h

Debbasch, F., and Rivet, J. P., 2010, "The Ludwig-Soret effect and stochastic processes", Journal of Chemical Thermodynamics, 43, 300306.

http://dx.doi.org/10.1016/j.jct.2010.09.010

Debuschewitz, C., and Köhler, W., 2001, "Molecular origin of thermal diffusion in benzene+ cyclohexane mixtures," Physical Review Letters, 87, 055901 .

http://dx.doi.org/10.1103/PhysRevLett.87.055901

De Gans, B-J., Kita, R., Wiegand, S., and Luettmer-Strathmann, J., 2003, "Unusual thermal diffusion in polymer solutions," Physical Review Letters, 91, 245501.

http://dx.doi.org/10.1103/PhysRevLett.91.245501

De Groot, S. R., and Mazur, P., 1984, "Non-Equilibrium Thermodynamics," Dover Publishers, New York.

Demirel, Y., and Sandler, S. I., 2001, "Linear-nonequilibrium thermodynamics theory for coupled heat and mass transport," International Journal of Heat and Mass Transfer, 44, 2439-2451. http://dx.doi.org/10.1016/S0017-9310(00)00291-X

Dominguez, G., Wilkins, G., and Thiemens, M. H., 2011, "The Soret effect and isotopic fractionation in high-temperature silicate melts," Nature, 473, 70-73.

http://dx.doi.org/10.1038/nature09911

Dougherty Jr., E. L., and Drickamer, H. G., 1955a, "Thermal diffusion and molecular motion in liquids," Journal of Physical Chemistry, 59, 443-449.

http://dx.doi.org/10.1021/j150527a016

Dougherty Jr., E. L., and Drickamer, H. G., 1955b, A theory of thermal diffusion in liquids, Journal of Chemical Physics, 23, 295-309.

http://dx.doi.org/10.1063/1.1741957

Duhr, S., Arduini, S., and Braun, D., 2004, "Thermophoresis of DNA determined by microfluidic fluorescence," The European Physical Journal E, 15, 277-286.

http://dx.doi.org/10.1140/epje/i2004-10073-5

Duhr, S., and Braun, D., 2006, "Why molecules move along a temperature gradient," Proceedings of the National Academy of Sciences, 103, 19678-19682.

http://dx.doi.org/10.1073/pnas.0603873103

Emery, A. H., and Drickamer, H. G., 1955, "Thermal diffusion in polymer solutions," Journal of Chemical Physics, 23, 2252.

http://dx.doi.org/10.1063/1.1740733

Eslamian, M., and Saghir, M. Z., 2009a, "Dynamic thermodiffusion model for binary liquid mixtures," Physical Review E, 80, 011201. http://dx.doi.org/10.1103/PhysRevE.80.011201 
Eslamian, M., and Saghir, M. Z., 2009b, "A critical review of thermodiffusion models: Role and significance of the heat of transport and the activation energy of viscous flow", Journal of Non-Equilibrium Thermodynamics, 34, 97-131.

http://dx.doi.org/10.1515/JNETDY.2009.007

Eslamian, M., and Saghir, M. Z., 2009c, "Microscopic study and modeling of thermodiffusion in binary associating mixtures," Physical Review E, 80, 061201.

http://dx.doi.org/10.1103/PhysRevE.80.061201

Eslamian, M., Sabzi, F., and Saghir, M. Z., 2010a, "Modeling of thermodiffusion in liquid metal alloys," Physical Chemistry Chemical Physics, 12, 13835-13848.

http://dx.doi.org/10.1039/c0cp00781a

Eslamian, M., Saghir, M. Z., and Bouali, M. M., 2010b, "Iinvestigation of the Soret effect in binary, ternary and quaternary hydrocarbon mixtures: New expressions for thermodiffusion factors in quaternary mixtures," International Journal of Thermal Sciences, 49, 2128-2137. http://dx.doi.org/10.1016/j.ijthermalsci.2010.06.015

Eslamian, M., and Saghir, M. Z., 2010c, "Dynamic thermodiffusion theory for ternary liquid mixtures," Journal of Nonequilibrium Thermodynamics, 35, 51.

http://dx.doi.org/10.1515/JNETDY.2010.004

Eslamian, M., and Saghir, M. Z., 2010d, "Non-equilibrium thermodynamic model for the estimation of the Soret coefficient in dilute polymer solutions," International Journal of Thermophysics, 32, 652-664.

http://dx.doi.org/10.1007/s10765-010-0898-2

Eslamian, M., and Saghir, M. Z., 2011, “Thermodiffusion (thermomigration) and convection in molten semiconductor-metal layers," International Journal of Thermal Sciences, 50, 1232-1242. http://dx.doi.org/10.1016/j.ijthermalsci.2011.02.014

Eslamian, M., and Saghir, M. Z., 2012a, "Thermodiffusion modeling of binary and ternary nonpolar hydrocarbon + alcohol mixtures", Journal of Nonequilibrium Thermodynamics, Under Review.

Eslamian, M., Jiang, C. G., and Saghir, M. Z., 2012b, "Role of the velocity frame of reference in thermodiffusion in liquid mixtures, Philosophical Magazine, In Press.

http://dx.doi.org/10.1080/14786435.2011.634845

Eslamian, M., and Saghir, M. Z., 2012c, "Estimation of thermodiffusion coefficients in ternary associating mixtures," Canadian Journal of Chemical Engineering, In Press.

http://dx.doi.org/10.1002/cjce.20581

Eslamian, M., and Saghir, M. Z., 2012d, "Thermodiffusion applications in MEMS, NEMS and solar cell fabrication by thermal metal doping of semiconductors," Fluid Dynamics and Material Processing, In Press

Eslamian, M., and Saghir, M. Z., 2012e, "Modeling of DNA thermophoresis in dilute solutions using the non-equilibrium thermodynamics approach", Journal of Nonequilibrium Thermodynamics, In Press.

http://dx.doi.org/10.1515/JNETDY.2011.026

Faissat, B. Knudsen, K., Stenby, E. H. and Montel, F., 1994, "Fundamental Statements about Thermal Diffusion for a Multicomponent Mixture in a Porous Medium," Fluid Phase
Equilibria, 100, 209-222.

http://dx.doi.org/10.1016/0378-3812(94)80010-3

Farago, J., Rousseau, B., and Artola, P-A. 2006. "On a variational approach to the Soret coefficient", Journal of Chemical Physics, 125, 164508

http://dx.doi.org/10.1063/1.2364499

Firoozabadi, A., Ghorayeb, and K, Shukla, K., 2000, "Theoretical model of thermal diffusion factors in multicomponent mixtures," AIChE Journal, 46, 892-900.

http://dx.doi.org/10.1002/aic.690460504

Gaeta, F. S., 1969, "Radiation pressure theory of thermal diffusion in liquids," Physical Review, 182, 289-296.

http://dx.doi.org/10.1103/PhysRev.182.289

Gautier, G., Ventura, L., Jérisian, R., Kouassi, S., Leborgne, C., Morillon, B., and Roy, M. 2006, "Deep trench etching combining aluminum thermomigration and electrochemical silicon dissolution," Applied Physics Letters, 88, 212501. http://dx.doi.org/10.1063/1.2206120

Galliero, G., and Montel, F., 2008, "Nonisothermal gravitational segregation by molecular dynamics simulation," Physical Review E, 78, 041203 .

http://dx.doi.org/10.1103/PhysRevE.78.041203

Gerl, M., 1967., "Contribution au calcul des forces agissant sur une impurete d'un metal soumis a un gradient de temperature," Journal of Physics and Chemistry of Solids, 28, 725-736.

http://dx.doi.org/10.1016/0022-3697(67)90004-2

Glasstone, S. Laidler, K. J., and Eyring, H., 1941, "The Theory of Rate Processes. The Kinetics of Chemical Reactions, Viscosity, Diffusion and Electrochemical Phenomena," McGraw-Hill Book Company New York - London.

Gonzalez-Bagnoli, M. G., Shapiro, A. A., and Stenby, E. E. 2003, "Evaluation of the thermodynamic models for the thermal diffusion factor," Philosophical Magazine, 83, 2171-2183. http://dx.doi.org/10.1080/0141861031000113280

Gusev, V., Wu, B., and Diebold, G. J., 2011, "Dynamics of thermal diffusion in a linear temperature field," Journal of Applied Physics, 110, 044908 .

http://dx.doi.org/10.1063/1.3625253

Haase, R., 1969, "Thermodynamics of Irreversible Processes," Addison-Wesley, Reading MA.

Hartung, M., Rauch, J., and Köhler, W., 2006, "Thermal diffusion of dilute polymer solutions: The role of solvent viscosity," Journal of Chemical Physics, 125, 214904.

http://dx.doi.org/10.1063/1.2393230

Henry, D., and Roux, B., 1986, "Three-dimensional numerical study of convection in a cylindrical thermal diffusion cell: Inclination effect," Physics of Fluids, 29, 3562-3572.

http://dx.doi.org/10.1063/1.865834

Iacopini, S., Rusconi, R., and Piazza, R., 2006, "The "macromolecular tourist": Universal temperature dependence of thermal diffusion in aqueous colloidal suspensions," The European Physical Journal E, 19, 
59-67.

http://dx.doi.org/10.1140/epje/e2006-00012-9

Jaber, T. J., Khawaja M., and Saghir M.Z. 2006, "Thermodiffusion of hydrocarbon binary mixtures in porous media," Fluid Dynamics and Material Processing, 2, 271-286.

Kempers, L. J. T. M., 1989, "A thermodynamic theory of the Soret effect in a multicomponent liquid," Journal of Chemical Physics, 90, 6541

http://dx.doi.org/10.1063/1.456321

Kempers, L. J. T. M., 2001, "A comprehensive thermodynamic theory of the Soret effect in a multicomponent gas, liquid, or solid," Journal of Chemical Physics, 115, 6330.

http://dx.doi.org/10.1063/1.1398315

Kincaid, J. M., Cohen, E. G. D., and Lopez de Haro, M., 1987, "The Enskog theory for multicomponent mixtures. IV. Thermal diffusion," Journal of Chemical Physics, 86, 963.

http://dx.doi.org/10.1063/1.452243

Kocherginsky, N., 2010, "Temperature-driven mass, charge and heat transfer in terms of physicochemical potential and Einstein's mobility," Chemical Engineering Science, 65, 4154-4159.

http://dx.doi.org/10.1016/j.ces.2010.04.035

Kolodner, P., Williams, H., and Moe, C., 1988, "Optical measurement of the Soret coefficient of ethanol/water solutions," Journal of Chemical Physics, 88, 6512.

http://dx.doi.org/10.1063/1.454436

Königer, A., Meier, B., and Köhler, W., 2009, "Measurement of the Soret, diffusion, and thermal diffusion coefficients of three binary organic benchmark mixtures and of ethanol/water mixtures using a beam deflection technique," Philosophical Magazine, 89, 907-923. http://dx.doi.org/10.1080/14786430902814029

Königer, A., Wunderlich, H., and Köhler, W., 2010, "Measurement of diffusion and thermal diffusion in ternary fluid mixtures using a twocolor optical beam deflection technique," Journal of Chemical Physics, 132, 174506

http://dx.doi.org/10.1063/1.3421547

Larre, J.P., Platten, J.K., and Chavepeyer, G. 1997, "Soret effects in ternary systems heated from below, International Journal of Heat and Mass Transfer, 40, 545-555.

http://dx.doi.org/10.1016/0017-9310(96)00125-1

Larson, R. G., Perkins, T. T., Smith, D. E., and Chu, S., 1997, "Hydrodynamics of a DNA molecule in a flow field," Physical Review $E, \mathbf{5 5}, 1794-1797$.

http://dx.doi.org/10.1103/PhysRevE.55.1794

Leahy-Dios, A., Bou-Ali, M. M. Patten, J. K., and Firoozabadi, A. 2005, "Measurements of molecular and thermal diffusion coefficients in ternary mixtures", Journal of Chemical Physics, 122, 234502. http://dx.doi.org/10.1063/1.1924503

Leahy-Dios, A, Zhuo, L., and Firoozabadi, A. 2008,"New thermal diffusion measurements for hydrocarbon binary mixtures: Viscosity and composition dependence," Journal of Physical Chemistry B, 112, 64426447.

http://dx.doi.org/10.1021/jp711090q
Legros, J.C., Longree, D., Chavepeyer, G., and Platten, J.K., 1975, "On the two-component Benard problem a numerical solution," Physica A, 80, $76-88$

http://dx.doi.org/10.1016/0378-4371(75)90147-8

Lozovskii, V. N., and Popov, V. P., 1983," Temperature gradient zone melting," Progress in Crystal Growth and Characterization, 6, 1-23. http://dx.doi.org/10.1016/0146-3535(83)90022-9

Luettmer-Strathmann, J., 2005, "Lattice model for thermodiffusion in polymer solutions," International Journal of Thermophysics, 26, 1693 1707.

http://dx.doi.org/10.1007/s10765-005-8589-0

Malmejac, Y., and Praizey, J. P., 1984, "Thermomigration of cobalt in liquid tin- Experiment 1 ES 320- In: Proc. Third European Symposium on Material Sciences in Space, Grenoble France, ESA SP-191, 127132.

Martin, A. and Bou-Ali, M. M., 2011,'Determination of thermal diffusion coefficient of nanofluid: Fullerene-toluene," C. R. Mecanique, 339, 329-334.

http://dx.doi.org/10.1016/i.crme.2011.03.010

Mes, E. P. C., Kok, W. T., and Tijssen, R., 2003, "Prediction of polymer thermal diffusion coefficients from polymer-solvent interaction parameters: comparison with thermal field flow fractionation and thermal diffusion forced Rayleigh scattering experiments," International Journal of Polymer Analysis and Characterization, 8, 133-153. http://dx.doi.org/10.1080/10236660304888

Morozov, K. I., 2009, "Soret effect in molecular mixtures," Physical Review E, 79, 031204.

http://dx.doi.org/10.1103/PhysRevE.79.031204

Nieto-Draghi, Ávalos, J. B., and Rousseau, B., 2005, "Computing the Soret coefficient in aqueous mixtures using boundary driven nonequilibrium molecular dynamics," Journal of Chemical Physics, 122, 114503.

http://dx.doi.org/10.1063/1.1863872

Ning, H., and Wiegand, S., 2006, "Experimental investigation of the Soret effect in acetone/water and dimethylsulfoxide/water mixtures," Journal of Chemical Physics, 125, 221102.

http://dx.doi.org/10.1063/1.2402159

Normann, R. A., Rousche, P. J., Horch, K. W., and Schmidt, S. P., 1994, "Impact inserter mechanism for implantation of a biomedical device," US Patent, US005361760A.

Otto, A., 1969, "Isotope separation by thermal diffusion in liquid metal," Science, 164, 297.

http://dx.doi.org/10.1126/science.164.3877.297

Perronace, A., Leppla, C., Leroy, F., Rousseau, B., and Wiegand, S., 2002, Soret and mass diffusion measurements and molecular dynamics simulations of n-pentane-n-decane mixtures, Journal of Chemical Physics, 116, 3718-3729.

http://dx.doi.org/10.1063/1.1436473

Pfann, W.G. 1969, “Zone melting,” US Patent, US3423189. 
Platten, J. K., 2006, "The Soret effect: a review of recent experimental results", Journal of Applied Mechanics, 73, 5-15.

http://dx.doi.org/10.1115/1.1992517

Platten, J. K., Marcoux, M., and Mojtabi, A., 2007, The RayleighBenard problem in extremely confined geometries with and without the Soret effect, C. R. Mecanique, 335, 638-654.

http://dx.doi.org/10.1016/i.crme.2007.08.011

Platten, J. K., Bou-Ali, M. M., Blanco, P., Madariaga, J. A., and Santamaria, C., 2007, "Soret coefficients in some water-methanol, water-ethanol, and water-isopropanol systems," The Journal of Physical Chemistry, 111, 11524-11530.

http://dx.doi.org/10.1021/jp074206z

Polyakov, P., Luettmer-Strathmann, J., and Wiegand, S., 2006, "Study of the thermal diffusion behavior of alkane/benzene mixtures by thermal diffusion forced Rayleigh scattering experiments and lattice model calculations," Journal of Physical Chemistry B, 110, 2621526224.

http://dx.doi.org/10.1021/jp065825v

Polyakov, P., and Wiegand, S., 2008, "Systematic study of the thermal diffusion in associated mixtures," Journal of Chemical Physics, 128, 034505 .

http://dx.doi.org/10.1063/1.2819672

Polyakov, P., Rossinsky, E., and Wiegand, S., 2009, "Study of the Soret effect in hydrocarbon chain/aromatic compound mixtures," Journal of Physical Chemistry E, 113, 13308-13312.

http://dx.doi.org/10.1021/jp904667p

Prager, S., and Eyring, H., 1953, "Thermal diffusion in binary systems", Journal of Chemical Physics, 21, 1347-1350.

http://dx.doi.org/10.1063/1.1699218

Praizey, J. P., 1986, "Thermomigration in liquid metallic alloys, Advances in Space Research, 6, 51-60.

http://dx.doi.org/10.1016/0273-1177(86)90183-3

Praizey, J. P., 1989, "Benefits of microgravity for measuring thermotransport coefficients in liquid metallic alloys, International Journal of Heat and Mass Transfer, 32, 2385-2401.

http://dx.doi.org/10.1016/0017-9310(89)90199-3

Praizey, J. P., Van Vaerenbergh, S., and Garandet, J. P., 1995, "Thermomigration experiment on board EURECA," Advances in Space Research, 16, 205-214.

http://dx.doi.org/10.1016/0273-1177(95)00161-7

Prigogine, I., de Brouckere, L., and Amand, R., 1950, "Recherches Sur La Thermodiffusion En Phase Liquide," Physica, 16, 851-860.

http://dx.doi.org/10.1016/0031-8914(50)90094-2

Reineck, P., Wienken, C. J. and Braun, D. 2010, "Thermophoresis of single stranded DNA,” Electrophoresis, 31, 279-286.

http://dx.doi.org/10.1002/elps.200900505

Rousseau, B., Nieto-Draghi, C., and, Avalos, J. B., 2004, "The role of molecular interactions in the change of sign of the Soret coefficient," Europhysics Letters, 67, 976-982.

http://dx.doi.org/10.1209/epl/i2004-10136-3

Rudolph, P., Boeck, T., and Schmidt, P., 1996," Thermodiffusion and morphological stability in convectionless crystal growth systems from melts and melt-solutions," Crystal Research and Technology, 31, 221229.

http://dx.doi.org/10.1002/crat.2170310218

Runyon, J. R., and Williams, S. K., 2011, "A theory-based approach to thermal field-flow fractionation of polyacrylates,"Journal of Chromatography A, 1218, 7016-7022. http://dx.doi.org/10.1016/i.chroma.2011.08.007

Rutherford, W. M., and Roof, J. G., 1959, "Thermal diffusion in methane-n-butane mixtures in the critical region," Journal of Physical Chemistry, 63, 1506-1511.

http://dx.doi.org/10.1021/j150579a045

Ryzhkov, I. I., and Shevtsova, V. M. 2009, "Long-wave instability of a multicomponent fluid layer with the Soret effect, Physics of Fluids, 21, 014102

http://dx.doi.org/10.1063/1.3054154

Samarao, A. K., and Ayazi, F., 2009, "Temperature compensation of silicon micromechanical resonators via degenerate doping," IEEE International Electron Devices Meeting (IEDM09), 789- 792.

Samarao, A. K., Casinovi, G., Ayazi, F., 2010, "Passive TCF compensation in high Q silicon micromechanical resonators," IEEE International Conference on Micro Electro Mechanical Systems, 116119.

Schimpf, M. E., and Giddings, J. C., 1989, "Characterization of thermal diffusion in polymer solutions by thermal field-flow fractionation: Dependence on polymer and solvent parameters, Journal of Polymer Science Part B: Polymer Physics, 27, 1317-1332.

http://dx.doi.org/10.1002/polb.1989.090270610

Semenov, S. N., 2010, "Statistical thermodynamic expression for the Soret coefficient," Europhysics Letters, 90, 56002. http://dx.doi.org/10.1209/0295-5075/90/56002

Semenov, S. N., and Schimpf, M. E., 2004, "Thermophoresis of dissolved molecules and polymers: Consideration of the temperatureinduced macroscopic pressure gradient," Physical Review E, 69, 011201.

http://dx.doi.org/10.1103/PhysRevE.69.011201

Semenov, S. N., and Schimpf, M. E., 2005, "Molecular thermodiffusion (thermophoresis) in liquid mixtures," Physical Review E, 72, 041202.

http://dx.doi.org/10.1103/PhysRevE.72.041202

Semenov, S. N., and Schimpf, M. E. 2009, "Mass transport thermodynamics in nonisothermal molecular liquid mixtures," PhysicsUspekhi, 52, 1045-1054.

http://dx.doi.org/10.3367/UFNe.0179.200910d.1105

Semenov, S., and Schimpf, M. E., 2011, "Internal degrees of freedom, molecular symmetry and thermodiffusion," C. R. Mecanique, 339, 335341 .

http://dx.doi.org/10.1016/j.crme.2011.03.011

Shapiro, A. A., 2004, "Fluctuation theory for transport properties in multicomponent mixtures: thermodiffusion and heat conductivity," Physica A, 332, 151-175.

http://dx.doi.org/10.1016/j.physa.2003.10.014

Shevtsova, V., Mialdun, A., Melnikov, D., Ryzhkov, I., Gaponenko, Y., Saghir, Z., Lyubimova, T., and Legros, J. C., 2011, "The IVIDIL experiment onboard the ISS: Themrodiffusion in the presence of controlled vibrations," C. R. Mecanique, 339, 310-317.

http://dx.doi.org/10.1016/j.crme.2011.03.007 
Shevtsova, V, Melnikov, D., Legros, J.C., Yan, Y, Saghir, M. Z., Lyubimova, T., Sedelnikov,G., and Roux, B., 2007, "Influence of vibrations on thermodiffusion in binary mixture: a benchmark of numerical solutions", Physics of Fluids, 19, 017111.

http://dx.doi.org/10.1063/1.2409622

Shukla, K., and Firoozabadi, A., 1998, "A new model of thermal diffusion coefficients in binary hydrocarbon mixtures," Industrial and Engineering Chemistry Research, 37, 3331-3343.

http://dx.doi.org/10.1021/ie970896p

Srinivasan, S., and Saghir, M. Z., 2010, "Thermo-Solutal-Diffusion In High Pressure Liquid Mixtures In the Presence of Micro-Vibrations", International Journal of Thermal Sciences, 49, 1613-1624.

http://dx.doi.org/10.1016/j.ijthermalsci.2010.04.018

Srinivasan, S., and Saghir, M. Z., 2011, "Experimental approaches to study thermodiffusion - A review," International Journal of Thermal Sciences, 50, 1125-1137.

http://dx.doi.org/10.1016/j.ijthermalsci.2011.02.022

Srinivasan, S., and Saghir, M. Z., 2012, "Modeling of thermotransport phenomenon in metal alloys using artificial neural networks, “ Applied Mathematical Modeling, Under Review.

Stadelmaier, D., and Köhler, W., 2009, "Thermal diffusion of dilute polymer solutions: The role of chain flexibility and the effective segment size," Macromolecules, 42, 9147-9152.

http://dx.doi.org/10.1021/ma901794k

Stadelmaier, D., and Köhler, W., 2008, From Small Molecules to High Polymers: Investigation of the Crossover of Thermal Diffusion in Dilute Polystyrene Solutions, Macromolecules, 41, 6205-6209.

http://dx.doi.org/10.1021/ma800891p

Sugisaki, M., Idemitsu, K., Mukai, S., and Furuya, H., 1981, "Thermal diffusion of tritium in $\mathrm{Nb}$ metal," Journal of Nuclear Materials, 104, 1493-1497.

http://dx.doi.org/10.1016/0022-3115(82)90811-X

Tichacek, L. J., Kmak, W. S., and Drickamer, H. G., 1956, "Thermal diffusion in liquids; the effect of non-ideality and association," Journal of Physical Chemistry, 60, 660-665.

http://dx.doi.org/10.1021/i150539a038

Tiller, W. A., 1963, "Migration of a liquid zone through a solid: Part I," Journal of Applied Physics, 34, 2757-2762.

http://dx.doi.org/10.1063/1.1729806

Van Vaerenbergh, S., Garandet, J. P., Praizey, J. P., and Legros, J. C., 1998, "Reference Soret coefficients of natural isotopes and diluted alloys of tin, "Physical Review E, 58, 1866-1873.

http://dx.doi.org/10.1103/PhysRevE.58.1866

Van Vaerenbergh, S., Srinivasan, S., and Saghir, M. Z., 2009, "Thermodiffusion in multi-component hydrocarbon mixtures:
Experimental investigations and computational analysis", Journal of Chemical Physics, 131, 114505.

http://dx.doi.org/10.1063/1.3211303

Villain-Guillot, S., and Würger, A. 2011, "Thermal diffusion in a binary liquid due to rectified molecular fluctuations," Physical Review $E, \mathbf{8 3}, 030501(\mathrm{R})$

http://dx.doi.org/10.1103/PhysRevE.83.030501

Wernick, J. H., 1956, "Determination of diffusivities in liquid metals by means of temperature gradient zone melting", Journal of Chemical Physics, 25, 47-49.

http://dx.doi.org/10.1063/1.1742846

Whitmore, F. C., 1960, "Experimental study of thermal diffusion in dilute solutions of high polymers," Journal of Applied Physics, 31, $1858-1864$

http://dx.doi.org/10.1063/1.1735464

Wiegand, S., 2004, "Thermal diffusion in liquid mixtures and polymer solutions," Journal of Physics: Condensed Matter, 16, R357.

http://dx.doi.org/10.1088/0953-8984/16/10/R02

Wiegand, S., Ning, H., and Kita, R., 2007, "Universal concentration dependence of the Soret coefficient in aqueous systems," Journal of Non-Equilibrium Thermodynamics, 32, 193-201.

http://dx.doi.org/10.1515/JNETDY.2007.011

Winter, F. R., and Drickamer, H. G., 1955," Thermal diffusion in liquid metals, Journal of Chemical Physics, 59, 1229-1230.

http://dx.doi.org/10.1021/j150534a011

Yan, Y., Blanco, P., Saghir, M. Z., and Bou-Ali, M. M., 2008, "An improved theoretical model for thermal diffusion coefficient in liquid hydrocarbon mixtures: comparison between experimental and numerical results," Journal of Chemical Physics, 129, 194507. http://dx.doi.org/10.1063/1.2976012

Yan, Y., Shevtsova, V., and Saghir, M. Z., 2005, "Numerical study of low frequency g-jitter effect on thermal diffusion", Fluid Dynamics and Material Processing, 1, 315-328.

Zebib, A., 2007, "Convective instabilities in thermogravitational columns," Journal of Nonequilibrium Thermodynamics, 32, 211-219. http://dx.doi.org/10.1515/JNETDY.2007.013

Zhang, M., and Müller-Plathe, F., 2006, "The Soret effect in dilute polymer solutions: Influence of chain length, chain stiffness, and solvent quality," Journal of Chemical Physics, 125, 124903.

http://dx.doi.org/10.1063/1.2356469

Zimmermann, G., Müller, U., and Davis, S. H., 1992, "Bénard convection in binary mixtures with Soret effects and solidification," Journal of Fluids Mechanics, 238, 657-682.

http://dx.doi.org/10.1017/S002211209200185X 